\title{
Cohomology and connections on fiber bundles and applications to field theories
}

Cite as: Journal of Mathematical Physics 37, 6375 (1996); https://doi.org/10.1063/1.531783

Submitted: 12 March 1996. Accepted: 04 June 1996 . Published Online: 04 June 1998

Christian Gross

\section{ARTICLES YOU MAY BE INTERESTED IN}

Gauge transformations and fiber bundle theory

Journal of Mathematical Physics 32, 2522 (1991); https://doi.org/10.1063/1.529146

Differential geometry, fiber bundles and physical theories

Physics Today 35, 41 (1982); https://doi.org/10.1063/1.2914967

Historical note on fiber bundles

Physics Today 68, 11 (2015); https://doi.org/10.1063/PT.3.2799

\begin{tabular}{|ll|l|}
\hline AVS Quantum Science & $\begin{array}{l}\text { A high impact interdisciplinary } \\
\text { journal for ALL quantum science }\end{array}$ & ACCEPTING SUBMISSIONS \\
AIP
\end{tabular}




\title{
Cohomology and connections on fiber bundles and applications to field theories
}

\author{
Christian Gross \\ Fachbereich Mathematik, TH Darmstadt, Germany
}

(Received 12 March 1996; accepted for publication 4 June 1996)

\begin{abstract}
Given any connection on a fiber bundle $B(M, F, G)$, we discuss the question which closed differential forms on the fiber $F$ can be extended to closed forms on the whole bundle $B$ in a canonical way such that the horizontal parts of the extended forms are given in terms of the gauge fields. Such questions arise for many models in theoretical physics such as the skyrmion bundle and related theories with nonAbelian groups $G$. We introduce the notion of a $G$-transgressive form as a sufficient condition for the form to be extendable to any bundle which comes along with a given left action $L: G \times F \rightarrow F$. Using Lie algebra cohomology we prove that if the structure group $G$ of the bundle is semisimple, then every closed $n$-form, $n \leqslant 2$, on the fiber which is invariant under $L$ is $G$-transgressive and thus defines a unique de Rham cohomology class on any bundle which comes along with $L$.

(C) 1996 American Institute of Physics. [S0022-2488(96)02011-7]
\end{abstract}

\section{MOTIVATION}

In theoretical physics the following general problem quite often occurs: suppose a field theory is defined for some manifold $F$, i.e., we have matter fields described by differentiable maps $f: M \rightarrow F$ from space-time $M$ to the manifold $F$. (Examples are wide-spread, let us only mention case of Dirac spinors, where $F=\mathrm{C}^{4}$, or the Skyrme model and related theories, where $F=\mathrm{SU}_{n}$, cf. Skyrme, ${ }^{1}$ Witten, ${ }^{2}$ and Zahed and Brown. ${ }^{3}$ ) For computations one has integrals like the action integral, where the Lagrangian is combined with the volume form of space-time, but also integrals over closed differential forms $\phi$ on $F$ (i.e., $d \phi=0$ ), which are integrated over space-time by means of the pullbacks $f^{\star}$.

Next suppose, we also have a symmetry group $G$ and we gauge our field theory with respect to this Lie group. From the mathematicians point of view, one has to construct a fiber bundle $B$ with base manifold $M$, (global) projection $\pi: B \rightarrow M$, (standard) fiber $F$ and structure group $G$, that acts on $F$ via an effective left action $L: G \times F \rightarrow F$, cf. Steenrod ${ }^{4}$ or Kobayashi and Numizu. ${ }^{5}$ For a cover $\mathfrak{U}=\left\{U_{\alpha}\right\}_{\alpha \in A}$ of the base manifold $M$, we have a bundle atlas $\left\{\left(U_{\alpha}, \psi_{\alpha}\right)\right\}_{\alpha \in A}$ with local trivializations $\psi_{\alpha}: \pi^{-1}\left(U_{\alpha}\right) \rightarrow U_{\alpha} \times F$, local projections $\pi_{\alpha}=\operatorname{pr}_{F} \circ \psi_{\alpha}$ onto $F$ and local injections $i_{\alpha, x}=\left(\pi_{\alpha, x}\right)^{-1}$ of $F$ onto the fiber over $x \in M$, where $\pi_{\alpha, x}:=\left.\pi_{\alpha}\right|_{\pi^{-1}(x)}: \pi^{-1}(x) \rightarrow F$. For every $f \in F$ and $g \in G$ define $L^{f}: G \rightarrow F$ and $L_{g}: F \rightarrow F$ by $L^{f}(g)=L_{g}(f)=L(g, f)$. Then on every overlap region $U_{\alpha \beta}=U_{\alpha} \cap U_{\beta}$ the change of bundle charts is given by transition functions $g_{\alpha \beta}: U_{\alpha \beta}=U_{\alpha} \cap U_{\beta} \rightarrow G$, such that $\pi_{\alpha, x} \circ\left(\pi_{\beta, x}\right)^{-1}=\pi_{\alpha, x} \circ i_{\beta, x}=L_{g_{\alpha \beta}(x)}$ for all $x \in U_{\alpha \beta}$.

The bundle $B(M, F, G)$ is associated to a principal bundle $P(M, G)$, where the structure group acts on itself by left multiplication. We will use the same symbols $\pi, \psi_{\alpha}, \pi_{\alpha}$, etc., for the bundles $P$ and $B$. On the principle bundle we also have a free right action $R: P \times G \rightarrow P$ and a connection $\Gamma$ defined by a connection 1 -form $\omega^{\Gamma}$ and its exterior covariant derivative, the curvature 2-form $\Omega^{\Gamma}$. Let $e$ denote the neutral element of $G$ and $\sigma_{\alpha, e}: U_{\alpha} \rightarrow \pi^{-1}\left(U_{\alpha}\right)=\left.P\right|_{U_{\alpha}}$ denote the local sections given by $\sigma_{\alpha, e}(x):=\psi_{\alpha}^{-1}(x, e)$. (Recall that a section $\sigma$ obeys $\pi \circ \sigma=\operatorname{id}_{M}$.) Then the gauge potentials $\mathrm{A}^{\alpha}$ and the gauge fields $\mathrm{F}^{\alpha}$ of the field theory are the local forms on the sets $U_{\alpha}$ defined by $\mathrm{A}^{\alpha}=\sigma_{\alpha, e}^{\star} \omega^{\Gamma}$, resp., $\mathrm{F}^{\alpha}=\sigma_{\alpha, e}^{\star} \Omega^{\Gamma}$.

Now the problem is as follows: since the matter fields now appear as global sections $f: M \rightarrow B$, it is necessary to "generalize" the given closed differential forms $\phi \in \mathscr{A}(F)$ to the bundle case (such that the pullbacks $f^{\star} \phi$ are well-defined): one needs a closed form $\psi \in \mathscr{C}(B)$ 
such that $\psi$ reproduces $\phi$ when restricted to the fibers: $\phi=i_{\alpha, x}^{\star} \psi$ for all $\alpha \in A$ and all $x \in U_{\alpha}$.

Recall that the de Rham cohomology of a manifold denotes the vector space of the closed forms modulo the exact forms on the manifold, i.e., it denotes the kernel of the exterior derivative $d: \mathscr{A}(M) \rightarrow \mathscr{A}(M), \mathscr{b}_{p-1}(M) \rightarrow \mathscr{C}_{p}(M)$ modulo its image. Since $d$ is a differential operator $(d \bigcirc d=0)$, all exact forms are closed and thus the vector space of the closed modulo the exact $p$-forms is well-defined. This quotient space is called the $p$-th de Rham cohomology group $H^{p}(M)$ and the (total) cohomology of $M$ means the direct sum $H^{*}(M):=\oplus_{p=0}^{\infty} H^{p}(M)$.

We may reformulate the problem in terms of the de Rham cohomology: Given a cohomology class $[\phi] \in H^{*}(F)$ we ask whether $[\phi]$ generates a cohomology class in $[\psi] \in H^{*}(B)$, such that $[\phi]=\left[i_{\alpha, x}^{\star}\right][\psi]$. Moreover, we also need a representative $\psi$ for this generated cohomology class.

The problem of computing the cohomology of a fiber bundle from $H^{*}(M)$ and $H^{*}(F)$ is a delicate mathematical problem. For a trivial bundle, i.e., a direct product $M \times F$, we have the Künneth formula

$$
H^{*}(M \times F) \cong H^{*}(M) \otimes H^{*}(F) .
$$

This formula is based on the fact that we have two global projections $\mathrm{pr}_{M}$ and $\mathrm{pr}_{F}$. Using their pullbacks we may extend any form on $M$ and $F$ to the bundle. Since $d$ commutes with pullbacks, this also holds for the cohomology classes. For a nontrivial bundle we only have one global projection $\pi$, which indeed allows us to lift any form on $M$ and any cohomology class in $H^{*}(M)$ onto the bundle. (Nevertheless, the induced homomorphism $\left[\pi^{*}\right]: H^{*}(M) \rightarrow H^{*}(B)$ needs not be injective nor surjective.) Yet there is no such mean for forms on $F$ and thus the situation becomes much more complicated as in the trivial case and leads to the theory of spectral sequences, cf. Bott and Tu. ${ }^{6}$ Spectral sequences compute $H^{*}(B)$ from $H^{*}(M)$ and $H^{*}(F)$. They also answer the question which closed forms on the fiber can be extended to closed forms on the bundle and thus generate a unique cohomology class in $H^{*}(B)$ in the manner above. We call these forms 0-transgressive. Not all closed forms on $F$ are 0-transgressive. In general it will depend on the structure of the bundle whether a given form is 0-transgressive: obviously for trivial bundles all closed forms on $F$ are 0 -transgressive.

If a form $\phi \in \mathscr{A}(F)$ is 0 -transgressive, spectral sequences also provide a formula for $\psi$ (Ref. 6, Prop. 9.5). Nevertheless this "Collating formula" involves a partition of unity subordinate to the given cover $\mathfrak{U}$ of $M$. For any such partition the formula gives a different form $\psi$ within the generated cohomology class. (Note that, a priori, $\psi$ is not unique but defined only up to an exact form on $B$, whose restriction to the fibers is zero.)

From the physicists point of view, this situation is quite unsatisfactory since a partition of unity does not bear any physical meaning and there is no reason why one partition - and the corresponding form $\psi$ - should be better than another. In fact one would like to obtain a representative $\psi$ for the generated cohomology class that can be associated with the physics in question, that is the gauge potentials and the gauge fields of the field theory.

This takes us back to connections on fiber bundles. Recall that a connection on a fiber bundle defines global horizontal and vertical projections of vector fields such that the $C^{\infty}(B)$-module $\mathscr{D}^{1}(B)$ of the vector fields on $B$ splits: $\mathscr{D}^{1}(B)=h \mathscr{D}^{1}(B) \oplus v \mathscr{D}^{1}(B)$. Once a connection on a principal bundle is defined via $\omega^{\Gamma}$, resp., the gauge potentials $\mathrm{A}^{\alpha}$, it also defines connections on all associated fiber bundles, cf. Section III.

In addition, $\Gamma$ defines lifts of vector fields on the base onto horizontal fields on the bundle and projections of forms on the bundle. These lifts and projections now can be used to extend forms on the fiber to the bundle. In fact, for every differential form $\phi \in \mathscr{A}(F)$ that is invariant under the given left action $L$ (i.e., $L_{g}^{\star} \phi=\phi$ for all $g \in G$ ), there exists exactly one vertical form on the bundle, say $\phi v \in \mathscr{C}(B)$, such that $\left.\phi v\right|_{\pi^{-1}(x)}=\phi$. From the physicists point of view, this seems to be a satisfactory generalization, but unfortunately we are not done with that, since the diagram in Figure 1 does not commute. 


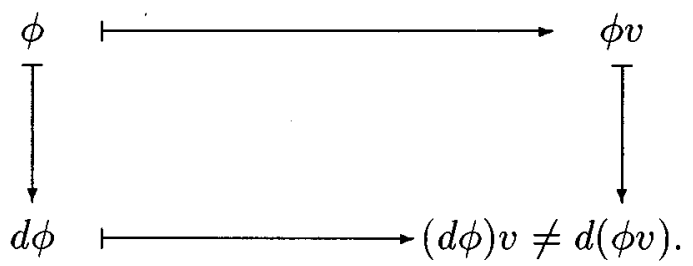

FIG. 1. Exterior derivative and vertical projection do not commute.

Thus although we start with a closed form $\phi$, the generated vertical form $\phi v$ needs not be closed. In general, we are not able to find a vertical representative for this cohomology class generated by a 0 -transgressive form, but we need to admit horizontal terms. Thus the question will be whether we can find such a representative where these horizontal terms are "naturally" given by the connection $\Gamma$, in fact, by the gauge fields. In that case, we call the resulting form adapted to $\Gamma$. Those forms are candidates for the desired generalizations of closed forms in field theories.

\section{BASIC DEFINITIONS}

Let $M$ denote any manifold and $V, W$ finite dimensional vector spaces. Every vector field $\mathscr{C} \in \mathscr{D}^{1}(M)$ differentiably associates with every $x \in M$ an element $\mathscr{C}_{x}$ in the tangent space $T_{x}(M)$. Now $\mathscr{D}_{p}(M, V)$, resp., $\mathscr{C}_{p}(M, V)$, denote the $C^{\infty}(M)$-modules of $p$-linear, resp., alternating $p$-linear, maps $\phi_{p}: \mathscr{D}^{1}(M) \times \cdots \times \mathscr{D}^{1}(M) \rightarrow C^{\infty}(M, V)$. They associate with every $x \in M$ an element $\phi_{x}=\left(\phi_{p}\right)_{x}$ in $\operatorname{Hom}\left(\otimes^{p} T_{x}(M), V\right)$, resp., in $\operatorname{Alt}_{p}\left(T_{x}(M), V\right)$, where $\operatorname{Alt}_{p}(W, V)$ means the vector space of all alternating $p$-linear maps from $W^{p}$ to $V$. The alternations $A_{p}: \mathscr{D}_{p}(M, V) \rightarrow \mathscr{D}_{p}(M, V)$ are the canonical projections of $\mathscr{D}_{p}(M, V)$ onto $\mathscr{C}_{p}(M, V)$. The exterior product (or wedge product) of forms will be denoted by $\wedge$ : for $\phi_{p} \in \mathscr{A}_{p}(M)$ and $\phi_{r}$ $\in \mathscr{A}_{r}(M)$ we have $\phi_{p} \wedge \phi_{r}:=A_{p+r}\left(\phi_{p} \otimes \phi_{r}\right) \in \mathscr{A}_{p+r}(M)$.

Also if a bilinear mapping $\varphi: V \times V \rightarrow V$ is given, $\wedge$ extends to an exterior product $\wedge_{\varphi}$ of $V$-valued differential forms. For $\phi=\hat{\phi} \otimes v \in \mathscr{C}(M) \otimes V \cong \mathscr{A}(M, V)$ and $\psi=\hat{\psi} \otimes w \in \mathscr{A}(M) \otimes V$, the exterior product is given by

$$
\phi \wedge_{\varphi} \psi:=(\hat{\phi} \wedge \hat{\psi}) \otimes \varphi(v, w)
$$

Everything in the sequel will also work for infinite dimensional vector spaces $V$, if we consider $\mathscr{b}(M) \otimes V$ instead of $\mathscr{C}(M, V)$. Yet for our purposes, we will restrict ourselves to finite dimensional $V$ and identify $\mathscr{A}(M) \otimes V$ and $\mathscr{A}(M, V)$.

As mentioned above, we are heading for differential forms whose horizontal parts are given in terms of the gauge fields. Let us denote the $C^{\infty}(B)$-module of horizontal forms on $B$ by $\mathscr{A}(B) h$ and the vertical counterpart by $\mathscr{A}(B) v$. (Note that only $\mathscr{C}_{1}(B)$ splits into $\mathscr{C}_{1}(B) h$ $\oplus \mathscr{C}_{1}(B) v$, whereas $\mathscr{C}_{0}(B) h=\mathscr{C}_{0}(B) v=\mathscr{C}_{0}(B)=C^{\infty}(B)$ and for $p \geqslant 2 \mathscr{C}_{p}(B)$ contains also "mixed" forms, i.e., exterior products of horizontal and vertical forms.) Normally one would try to split a form into a sum $\phi=\Sigma_{i \in I} \phi_{i}$, where the $\phi_{i}$ are given by $\phi_{i}=\phi_{i}^{1} \wedge \phi_{i}^{2}$ with $\phi_{i}^{1}$ $\in \mathscr{A}(B) h$ and $\phi_{i}^{2} \in \mathscr{A}(B) v$.

Yet in our case such a splitting is impossible, since the gauge fields and the gauge potentials are Lie algebra valued differential forms: $\mathrm{A}^{\alpha} \in \mathscr{b}_{1}\left(U_{\alpha}, \mathfrak{g}\right)$ and $\mathrm{F}^{\alpha} \in \mathscr{C}_{2}\left(U_{\alpha}, \mathfrak{g}\right)$, where $\mathfrak{g}$ denotes the Lie algebra of the structure group $G$. In contrast, $\phi \in \mathscr{A}(F)-$ and thus also $\psi \in \mathscr{A}(B)-$ will be real or complex valued. Thus we need a generalization of the wedge product that combines $\mathfrak{g}$-valued forms with $(\mathfrak{g} \otimes \cdots \otimes \mathfrak{g})^{*}$-valued forms in order to produce real or complex valued forms. This is the task of the following definition.

Definition II.1: For $\chi_{r}^{s} \in \mathscr{A}_{r}\left(M, \operatorname{Hom}\left(\otimes^{s} \mathfrak{g}, V\right)\right)$ and $\phi_{p} \in \mathscr{A}_{p}(M, \mathfrak{g}), p, r, s-1 \in \mathbb{N}_{0}$, let $d_{r+s p} \in \mathscr{D}_{r+s p}(M, V)$ with 


$$
\begin{gathered}
d_{r+s p}\left(\mathscr{C}^{1}, \ldots, \mathscr{C}^{r+s p}\right)(x):=\left[\chi_{x}\left(\mathscr{C}_{x}^{1}, \ldots, \mathscr{X}_{x}^{r}\right)\right] \bigcirc\left[\phi_{x}\left(\mathscr{C}_{x}^{r+1}, \ldots, \mathscr{X}_{x}^{r+p}\right)\right. \\
\left.\otimes \cdots \otimes \phi_{x}\left(\mathscr{C}_{x}^{r+(s-1) p+1}, \ldots, \mathscr{C}_{x}^{r+s p}\right)\right]
\end{gathered}
$$

for all $x \in M$ and define $\chi_{r}^{s} \bullet \phi_{p}:=A_{r+s p}\left(d_{r+s p}\right) \in \mathscr{A}_{r+s p}(M, V)$ and $\chi_{r}^{0} \bullet \phi_{p}:=\chi_{r}^{0}$.

Roughly speaking, the bullet operator means the following: for any $x \in M$ and $\mathscr{X}^{i}$ $\in \mathscr{D}^{1}(M), \chi_{x}\left(\mathscr{C}_{x}^{1}, \ldots, \mathscr{C}_{x}^{r}\right)$ defines an element in $\operatorname{Hom}\left(\otimes{ }^{s} \mathfrak{g}, V\right)$. Thus we need $s$ vectors in $\mathfrak{g}$ as input for this map. But again for any $x \in M$ and $\mathscr{F}^{i} \in \mathscr{D}^{1}(M), \phi_{x}\left(\mathscr{Y}_{x}^{1}, \ldots, \mathscr{Y}_{x}^{p}\right)$ defines such a vector in $\mathfrak{g}$. Altogether the combination of $\chi$ and $s$ factors $\phi$ defines an element $d_{r+s p}^{s q}$ $\in \mathscr{D}_{r+s p}(M, V)$. Using the alternation $A_{r+s p}$, we finally obtain a form in $\mathscr{C}_{r+s p}(M, V)$.

Note that this construction works with any finite dimensional vector space $W$ instead of $\mathfrak{g}$, and even with differential forms $\phi_{p}^{q} \in \mathscr{A}_{p}\left(M, \operatorname{Hom}\left(\otimes^{q} X, W\right)\right)$, where $X$ is another finite dimensional vector space. In the latter case the construction produces a differential form $\chi_{r}^{s} \cdot \phi_{p}^{q}$ $\in \mathscr{b}_{r+s p}\left(M, \operatorname{Hom}\left(\otimes^{s q} X, V\right)\right)$. Yet we only need $\bullet$ in the way defined above, especially for $V=\mathbb{R}, \mathrm{C}$ and $\chi_{r}^{s} \in \mathscr{C}_{r}\left(M, \operatorname{Sym}_{s}(\mathfrak{g}, V)\right)$, where $\operatorname{Sym}_{s}(\mathfrak{g}, V)$ denotes the vector space of symmetric $s$-linear maps from $\mathfrak{g}^{s}$ to $V$, i.e., the image of the canonical symmetrization $\operatorname{Sym}: \operatorname{Hom}\left(\otimes^{s} \mathfrak{g}, V\right) \rightarrow \operatorname{Hom}\left(\otimes^{s} \mathfrak{g}, V\right)$.

For vectors $E_{j} \in \mathfrak{g}, j=1, \ldots, s$, let $E_{1} \otimes \cdots \otimes E_{s}: \operatorname{Hom}\left(\otimes^{s} \mathfrak{g}, V\right) \rightarrow V$ denote the canonical (evaluation) morphism. For any form $\chi_{r}^{s} \in \mathscr{A}_{r}\left(M, \operatorname{Hom}\left(\otimes^{s} \mathfrak{g}, V\right)\right)$ define $\chi_{r}^{E_{1}}, \ldots, E_{s} \in \mathscr{A}_{r}(M, V)$ to be the push-out of $\chi_{r}^{s}$ under this morphism: $\chi_{r}^{E_{1}, \ldots, E_{s}}:=\left(E_{1} \otimes \cdots \otimes E_{s}\right)_{\star} \chi_{r}^{s}$ i.e., for all $x \in M$ and $\mathscr{B}^{i} \in \mathscr{D}^{1}(M), i=1, \ldots, r$,

$$
\begin{gathered}
\left(\chi_{r}^{E_{1}, \ldots, E_{s}}\right)_{x}\left(\mathscr{O}_{x}^{1}, \ldots, \mathscr{C}_{x}^{r}\right):=\left(E_{1} \otimes \cdots \otimes E_{s}\right) \circ\left(\chi_{r}^{s}\right)_{x}\left(\mathscr{X}_{x}^{1}, \ldots, \mathscr{C}_{x}^{r}\right) \\
=\left[\left(\chi_{r}^{s}\right)_{x}\left(\mathscr{C}_{x}^{1}, \ldots, \mathscr{C}_{x}^{r}\right)\right]\left(E_{1} \otimes \cdots \otimes E_{s}\right) .
\end{gathered}
$$

With this convention we obtain the following lemma:

Lemma II.2: Let $p, r, s-1 \in \mathbb{N}_{0}$. If $\phi_{p}=\sum_{i=1}^{m} \phi^{i} \otimes E_{i}$ with $\phi^{i} \in \mathscr{A}_{p}(M)$ and $E_{i} \in \mathfrak{g}$, then

$$
\chi_{r}^{s} \bullet \phi_{p}=\sum_{i_{1}, \ldots, i_{s}=1}^{m} \chi_{r}^{E_{i_{1}}, \ldots, E_{i_{s}}} \wedge \phi^{i_{1}} \wedge \ldots \wedge \phi^{i_{s}}
$$

Proof: Take $x \in M$ and $\mathscr{O}^{i} \in \mathscr{D}^{1}(M)$. Then for $d_{r+s p}$ in Definition II we obtain

$$
\begin{aligned}
& \left(d_{r+s p}\right)_{x}\left(\mathscr{C}_{x}^{1}, \ldots, \mathscr{X}_{x}^{r+s p}\right): \\
& =\left[\chi_{x}\left(\mathscr{C}_{x}^{1}, \ldots, \mathscr{C}_{x}^{r}\right)\right]\left(\phi_{x}\left(\mathscr{C}_{x}^{r+1}, \ldots, \mathscr{X}_{x}^{r+p}\right)\right. \\
& \left.\otimes \cdots \otimes \phi_{x}\left(\mathscr{C}_{x}^{r+(s-1) p+1}, \ldots, \mathscr{\mathscr { C }}_{x}^{r+s p}\right)\right) \\
& =\sum_{i_{1}, \ldots, i_{s}=1}^{m}\left[\chi_{x}\left(\mathscr{C}_{x}^{1}, \ldots, \mathscr{C}_{x}^{r}\right)\right]\left(\left(E_{i_{1}} \otimes \cdots \otimes E_{i_{s}}\right)\right. \\
& \left.\cdot \prod_{j=1}^{s} \phi_{x}^{i_{j}}\left(\mathscr{C}_{x}^{r+(j-1) p+1}, \ldots, \mathscr{X}_{x}^{r+j p}\right)\right) \\
& =\sum_{i_{1}, \ldots, i_{s}=1}^{m}\left[\left(\chi_{r}^{E_{i_{1}}, \ldots, E_{i_{s}}}\right)_{x}\left(\mathscr{C}_{x}^{1}, \ldots, \mathscr{C}_{x}^{r}\right)\right] \\
& \cdot \prod_{j=1}^{s} \phi_{x}^{i j}\left(\mathscr{X}_{x}^{r+(j-1) p+1}, \ldots, \mathscr{X}_{x}^{r+j p}\right) .
\end{aligned}
$$


Thus if $S_{r+s p}$ denotes the group of permutations of $r+s p$ elements and $(-1)^{\rho}$ means the signum of $\rho \in S_{r+s p}$, we get

$$
\begin{aligned}
& \left(\chi_{r}^{s} \bullet \phi_{p}\right)_{x}\left(\mathscr{C}_{x}^{1}, \ldots, \mathscr{C}_{x}^{r+s p}\right):=\left[A_{r+s p}\left(d_{r+s p}\right)\right]_{x}\left(\mathscr{C}_{x}^{1}, \ldots, \mathscr{C}_{x}^{r+s p}\right) \\
& =\sum_{\rho \in S_{r+s p}} \frac{(-1)^{\rho}}{(r+s p) !_{i_{1}}, \ldots, i_{s}=1} \sum^{m}\left[\left(\chi_{r}^{E_{i_{1}}, \ldots, E_{i_{s}}}\right)_{x}\left(\mathscr{O}_{x}^{\rho(1)}, \ldots, \mathscr{C}_{x}^{\rho(r)}\right)\right] \\
& \cdot \prod_{j=1}^{s} \phi_{x}^{i_{j}}\left(\mathscr{C}_{x}^{p(r+(j-1) p+1)}, \ldots, \mathscr{C}_{x}^{\rho(r+j p)}\right)
\end{aligned}
$$

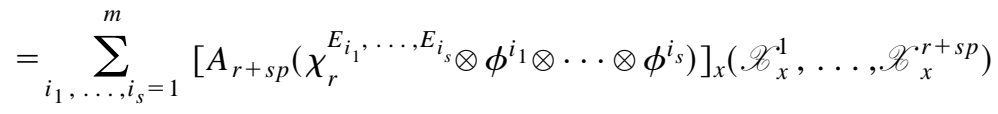

$$
\begin{aligned}
& =\left(\sum_{i_{1}, \ldots, i_{s}=1}^{m} \chi_{r}^{E_{i_{1}}, \ldots, E_{i_{s}}} \bigwedge \phi^{i_{1}} \wedge \ldots \wedge \phi^{i_{s}}\right)_{x}\left(\mathscr{C}_{x}^{1}, \ldots, \mathscr{C}_{x}^{r+s p}\right),
\end{aligned}
$$

where the last identity follows immediately from the definition of the exterior product $\wedge$.

Lemma II.2 proves that if $p$ is even, then only the symmetric part of $\chi_{r}^{s}$ counts: $\chi_{r}^{s} \bullet \phi_{p}=\left(\operatorname{Sym}_{\star} \chi_{r}^{s}\right) \bullet \phi_{p}$. Thus we may concentrate on $\chi_{r}^{s} \in \mathscr{A}_{r}\left(M, \operatorname{Sym}_{s}(\mathfrak{g}, V)\right)$, in order to construct forms $\chi_{r}^{s} \bullet \mathrm{F}$ with the 2-forms $\mathrm{F}^{\alpha}$.

Let $\operatorname{Ad}^{\star}: G \times \operatorname{Hom}\left(\otimes^{s} \mathfrak{g}, V\right) \rightarrow \operatorname{Hom}\left(\otimes^{s} \mathfrak{g}, V\right)$ denote the right representation induced by the adjoint action: for $K \in \operatorname{Hom}\left(\otimes^{s} \mathfrak{g}, V\right), g \in G$ and $E_{i} \in \mathfrak{g}$, it is defined by

$$
\left(\operatorname{Ad}(g)^{\star} K\right)\left(E_{1}, \ldots, E_{s}\right):=K\left(\operatorname{Ad}(g) E_{1}, \ldots, \operatorname{Ad}(g) E_{s}\right)
$$

A differential form $\chi_{n}^{s} \in \mathscr{C}_{n}\left(F, \operatorname{Hom}\left(\otimes^{s} \mathfrak{g}, V\right)\right)$ is called $\left(G\right.$-)equivariant if $L_{g}^{\star} \chi_{n}^{s}$ $=\left(\operatorname{Ad}\left(g^{-1}\right)^{\star}\right)_{\star} \chi_{n}^{s}$. (For $s=0$ this obviously means that $\chi_{n}^{0}$ is invariant under $L$.) On the other hand a differential form $\phi \in \mathscr{A}(F, \mathfrak{g})$ is called $\left(G\right.$-)equivariant if $L_{g}^{\star} \phi=\operatorname{Ad}(g)_{\star} \phi$, e.g., $\omega^{\Gamma}$ $\in \mathscr{b}_{1}(P, \mathfrak{g})$ and $\Omega^{\Gamma} \in \mathscr{b}_{2}(P, \mathfrak{g})$ are equivariant, since $R_{g}^{\star} \omega^{\Gamma}=\operatorname{Ad}\left(g^{-1}\right)_{\star} \omega^{\Gamma}$, resp., $R_{g}^{\star} \Omega^{\Gamma}=\operatorname{Ad}\left(g^{-1}\right)_{\star} \Omega^{\Gamma}$. We denote the sets of these equivariant differential forms by $\mathscr{b}\left(F, \operatorname{Hom}\left(\otimes^{s} \mathfrak{g}, V\right)\right)_{\text {equiv }}$, resp., $\mathscr{b}(F, \mathfrak{g})_{\text {equiv }}$. They are modules of the exterior algebra $\mathscr{A}(P)_{\text {inv }}$ of invariant differential forms.

Lemma II.3: If $\chi_{n}^{s} \in \mathscr{A}_{n}\left(F, \operatorname{Hom}\left(\otimes^{s} \mathfrak{g}, V\right)\right)_{\text {equiv }}$ and $\phi_{p} \in \mathscr{A}_{p}(F, \mathfrak{g})_{\text {equiv }}$, then $\chi_{n}^{s} \bullet \phi_{p}$ is invariant.

For our purposes we also need further operators on differential forms that transform $V$-valued forms into $\operatorname{Alt}_{i}(\mathfrak{g}, V)$-valued forms. Recall that for any Lie group action $L: G \times F \rightarrow F$, every $X \in \mathfrak{g}$ canonically induces a vector field $\mathscr{L}_{X} \in \mathscr{D}^{1}(F)$ by $\left(\mathscr{L}_{X}\right)_{f}:=\left(d L^{f}\right)_{e}(X)$, where $\left(d L^{f}\right)_{e}: T_{e}(G) \rightarrow T_{f}(F)$ denotes the differential of the map $L^{f}$. The operator $-\mathscr{L}: \mathfrak{g} \rightarrow \mathscr{D}^{1}(F)$ is a Lie algebra homomorphism, in fact we have

$$
\begin{gathered}
{\left[\mathscr{L}_{X}, \mathscr{L}_{Y}\right]=\mathscr{L}_{[Y, X]}=-\mathscr{L}_{[X, Y]} \text { for all } X, Y \in \mathfrak{g},} \\
\left(L_{g}\right)_{\star} \mathscr{L}_{X}=\mathscr{L}_{\operatorname{Ad}(g) X} \text { for all } g \in G, X \in \mathfrak{g} .
\end{gathered}
$$

Analogously for the right action $R$ on a principal bundle, $\mathscr{B}: \mathfrak{g} \rightarrow \mathscr{D}^{1}(P)$ is a Lie algebra homomorphism, and the $\mathscr{B}_{X}$ are the so-called fundamental vector fields on $P$. Now we may define:

Definition II.4: Let $L$ be a Lie group action of $G$ on $F$ and $\omega_{n} \in \mathscr{A}_{n}(F, V)$. We define differential forms $L_{\bullet}^{i} \omega_{n} \in \mathscr{A}_{n-i}\left(F, \operatorname{Alt}_{i}(\mathfrak{g}, V)\right), i=0, \ldots, n$, for all $\mathscr{C}^{j} \in \mathscr{D}^{\mathfrak{1}}(F), E_{k} \in \mathfrak{g}$ and $f$ $\in F$ by 
$\left[\left(L_{\bullet}^{i} \omega_{n}\right)\left(\mathscr{C}^{1}, \ldots, \mathscr{C}^{n-i}\right)(f)\right]\left(E_{1}, \ldots, E_{i}\right):=\frac{n !}{(n-i) !} \omega_{n}\left(\mathscr{C}^{1}, \ldots, \mathscr{C}^{i}, \mathscr{C}^{1}, \ldots, \mathscr{C}^{n-i}\right)(f) \in V$,

where $\mathscr{L}^{i}:=\mathscr{L}_{E_{i}}$. For $i>n$ we put $L_{\bullet}^{i} \omega_{n}=0$. In the case $i=1$ we also define for $\chi_{n}^{s}$ $\in \mathscr{A}_{n}\left(F, \operatorname{Hom}\left(\otimes{ }^{s} \mathfrak{g}, V\right)\right)$

$$
L_{\bullet} \chi_{n}^{s}:=\operatorname{Sym}_{\star}\left(L_{\bullet} \chi_{n}^{s}\right) \in \mathscr{A}_{n-1}\left(F, \operatorname{Sym}_{s+1}(\mathfrak{g}, V)\right) .
$$

$\left(\right.$ Obviously $\operatorname{Sym}_{\star}\left(L_{\bullet}^{i} \chi_{n}^{s}\right)=0$ for $i>1$.) Now if $\iota_{\mathscr{X}}: \mathscr{C}_{n}(M, V) \rightarrow \mathscr{C}_{n-1}(M, V)$ denotes the interior product with respect to $\mathscr{X}^{6} \in \mathscr{D}^{1}(M)$, which is given by

$$
\left(\iota_{\mathscr{X}} \omega_{n}\right)\left(\mathscr{Y}^{1}, \ldots, \mathscr{Y}^{n-1}\right):=n \omega_{n}\left(\mathscr{X}, \mathscr{Y}^{1}, \ldots, \mathscr{Y}^{n-1}\right),
$$

then we have the following relation with regard to our convention (2):

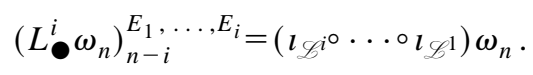

The following lemma is quite immediate by (4):

Lemma II.5: For all $i \leqslant n$, the operator $L_{\bullet}^{i}: \mathscr{A}_{n}(F, V) \rightarrow \mathscr{A}_{n-i}\left(F, \operatorname{Alt}_{i}(\mathfrak{g}, V)\right)$ is $C^{\infty}(F)$-linear. For $\omega_{n} \in \mathscr{A}_{n}(F, V)$ and $\chi_{n}^{s} \in \mathscr{C}_{n}\left(F, \operatorname{Hom}\left(\otimes^{s} \mathfrak{g}, V\right)\right)_{\text {equiv }}$ we have

$$
\begin{gathered}
L_{\bullet}^{0} \omega_{n}=\omega_{n}, \quad\left(L_{\bullet}^{n} \omega_{n}\right)(f)=n !\left[\left(L^{f}\right)^{\star} \omega_{n}\right]_{e} \text { for all } f \in F, \\
L_{g}^{\star}\left(L_{\bullet}^{i} \omega_{n}\right)=\left(\operatorname{Ad}\left(g^{-1}\right)^{\star}\right){ }_{\star}\left[L_{\bullet}^{i}\left(L_{g}^{\star} \omega_{n}\right)\right], \text { thus } \\
L_{g}^{\star}\left(L_{\bullet}^{i} \chi_{n}^{s}\right)=\left(\operatorname{Ad}\left(g^{-1}\right)^{\star}\right)_{\star}\left(L_{\bullet}^{i} \chi_{n}^{s}\right) \text { and } \quad L_{g}^{\star}\left(L_{\bullet}^{\vee} \chi_{n}^{s}\right)=\left(\operatorname{Ad}\left(g^{-1}\right)^{\star}\right)_{\star}\left(L_{\bullet} \chi_{n}^{s}\right) .
\end{gathered}
$$

Equation (7) yields that $L_{\bullet}^{i} \omega_{n}$ and $L_{\bullet}^{\vee} \omega_{n}$ are $G$-equivariant if $\omega_{n}$ is invariant under $L$.

\section{EXTENDING FORMS TO THE BUNDLE}

If $P$ is a principal bundle with free right Lie group action $R$, and $L$ is a left effective Lie group action of $G$ on a manifold $F$, then the associated fiber bundle with fiber $F$ that comes along with $L$ is the quotient manifold $B(M, F, G)=P \times{ }_{G} F$ of the direct product $P \times F$ under the free right action $\widetilde{R}:(P \times F) \times G \rightarrow P \times F$, which is defined by

$$
\widetilde{R}_{g}(p, f):=\left(R_{g}(p), L_{g^{-1}}(f)\right) \text { for all } p \in P, f \in F .
$$

In fact, $P \times F$ is a principal bundle over $B$ with projection $\widetilde{\pi}$ and fiber $G$. Every connection $\Gamma$ on a principal bundle, given by a connection 1-form $\omega^{\Gamma}$, canonically induces a connection $\widetilde{\Gamma}$ on $(P \times F)(B, G)$ by $\widetilde{\omega}^{\widetilde{\Gamma}}=\operatorname{pr}_{P}^{\star} \omega^{\Gamma}$. In turn, $\widetilde{\Gamma}$ defines horizontal lifts of vector fields $\widetilde{\mathrm{L}}: \mathscr{D}^{1}(B) \rightarrow \widetilde{h} \mathscr{D}^{1}(P \times F)_{\text {inv }}$. $\widetilde{\mathrm{L}}$ is a $C^{\infty}(B)$-module isomorphism with inverse morphism $d \widetilde{\pi}$. If $h^{\text {nat }}$ and $v^{\text {nat }}$ denote the natural projections of vector fields on the direct product $P \times F$, then we also have projections $h, v$ on $\mathscr{D}^{1}(B)$

$$
h=d \tilde{\pi} h^{\mathrm{nat}} \widetilde{\mathrm{L}}, \quad v=d \widetilde{\pi} v^{\mathrm{nat}} \widetilde{\mathrm{L}},
$$

so

$$
\mathscr{D}^{1}(B)=h \mathscr{D}^{1}(B) \oplus v \mathscr{D}^{1}(B) .
$$


This is the induced connection on the associated bundle $B$, as mentioned above. Let $\mathscr{C}^{i}$ $\in \mathscr{D}^{1}(B)$, then for any $\omega_{n} \in \mathscr{A}_{n}(B, V), n>0$, the horizontal and vertical projections $\omega_{n} h$ $\in \mathscr{A}_{n}(B, V) h$, resp., $\omega_{n} v \in \mathscr{C}_{n}(B, V) v$, are given by

$$
\begin{aligned}
& \omega_{n} h\left(\mathscr{C}^{1}, \ldots, \mathscr{C}^{n}\right):=\omega_{n}\left(h \mathscr{C}^{1}, \ldots, h \mathscr{C}^{n}\right), \\
& \omega_{n} v\left(\mathscr{C}^{1}, \ldots, \mathscr{C}^{n}\right):=\omega_{n}\left(v \mathscr{C}^{1}, \ldots, v \mathscr{C}^{n}\right) .
\end{aligned}
$$

Obviously these projections commute with •, i.e.,

$$
\left(\chi_{n}^{s} \bullet \phi_{p}\right) h=\chi_{n}^{s} h \cdot \phi_{p} h, \quad\left(\chi_{n}^{s} \bullet \phi_{p}\right) v=\chi_{n}^{s} v \bullet \phi_{p} v
$$

Recall that, by definition, $\omega^{\Gamma}$ is a vertical form. The exterior covariant derivative of forms on $P$ is defined by $d^{\Gamma} \phi:=(d \phi) h$. Thus $\Omega^{\Gamma}=d^{\Gamma} \omega^{\Gamma}=\left(d \omega^{\Gamma}\right) h$ is a horizontal form.

If $\phi$ is a differential form on $F$, then $\operatorname{pr}_{F}^{\star} \phi$ is a form on $P \times F$, and if $\mathscr{F}^{i}$ are vector fields on $B$, then $\left(\operatorname{pr}_{F}^{\star} \phi\right)\left(\ldots, \widetilde{\mathbb{L}} \mathscr{Y}^{i}, \ldots\right)$ is a function on $P \times F$. Now if $\phi$ is invariant under $L$, one can prove that this function is invariant under $\widetilde{R}$ and thus defines a map on $B$. But this defines a form on $B$. In fact, we have the following proposition: ${ }^{7}$

Proposition III.1: $\phi \in \mathscr{A}(F, V)$ defines a vertical $V$-valued form $\phi v$ on $B(M, F, G)$ iff $\phi$ is invariant under all $L_{g}^{\star}$ For such a $\phi$ and all $\mathscr{H}^{i} \in \mathscr{D}^{1}(B)$ then there exists $f \in C^{\infty}(B, V)$ with

$$
\left(\operatorname{pr}_{F}^{\star} \phi\right)\left(\ldots, \widetilde{L}^{i}{ }^{i}, \ldots\right)=f \bigcirc \widetilde{\pi}
$$

If $h^{\alpha}$ and $v^{\alpha}$ denote the local projections of fields and forms induced by $\Gamma$, then this generated form $\phi v$ locally is given by

$$
\left.\phi v\right|_{\pi^{-1}\left(U_{\alpha}\right)}=\left(\pi_{\alpha}^{\star} \phi\right) v^{\alpha}
$$

Proposition III.1 is a special case of the following theorem:

Theorem III.2: If $\chi_{n}^{s} \in \mathscr{A}_{n}\left(F, \operatorname{Hom}\left(\otimes^{s} \mathfrak{g}, V\right)\right)_{\text {equiv }}$ and $\phi \in \mathscr{A}_{p}(P, \mathfrak{g})_{\text {equiv }}, p \in \mathbb{N}_{0}$, then $\left(\operatorname{pr}_{F}^{\star} \chi_{n}^{s}\right) \bullet\left(\operatorname{pr}_{P}^{\star} \phi\right) \in \mathscr{C}_{n+s p}(P \times F, V)$ defines a $V$-valued form on $B$ : for all vector fields $\mathscr{F}^{i}$ $\in \mathscr{D}^{1}(B)$ then there exists $f \in C^{\infty}(B, V)$ such that

$$
\begin{aligned}
& {\left[\left(\operatorname{pr}_{F}^{\star} \chi_{n}^{s}\right) \bullet\left(\operatorname{pr}_{P}^{\star} \phi\right)\right]\left(\ldots, \widetilde{L} \mathscr{H}^{i}, \ldots\right)} \\
& \quad=\left[\left(\operatorname{pr}_{F}^{\star} \chi_{n}^{s}\right) \bullet\left(\operatorname{pr}_{P}^{\star} \phi h\right)\right]\left(\ldots, \widetilde{L} \mathscr{Y}^{i}, \ldots\right)=f \bigcirc \widetilde{\pi}
\end{aligned}
$$

$\left(\mathrm{pr}_{F}^{\star} \chi\right)$ defines the vertical and $\left(\operatorname{pr}_{P}^{\star} \phi\right)$ defines the horizontal part of the form.

The proof of Theorem III.2 relies on Lemma II.3: under the assumed conditions one shows that $\left(\operatorname{pr}_{F}^{\star} \chi_{n}^{s}\right)$ and $\left(\operatorname{pr}_{P}^{\star} \phi\right)$ are equivariant with respect to $\widetilde{R}$, and thus $\left(\operatorname{pr}_{F}^{\star} \chi_{n}^{s}\right) \bullet\left(\operatorname{pr}_{P}^{\star} \phi\right)$ is invariant by Lemma II.3.

Natural candidates for $\phi \in \mathscr{C}_{p}(P, \mathfrak{g})_{\text {equiv }}$ are $\omega^{\Gamma}$ and $\Omega^{\Gamma}$. Nevertheless, since $\omega^{\Gamma}$ is vertical, Theorem III.2 yields that the generated form on $B$ is zero. For $\Omega^{\Gamma}$, the generated form is locally given by

$$
\left[\left(\pi_{\alpha}^{\star} \chi_{n}^{s}\right) v^{\alpha}\right] \bullet\left(\pi^{\star} \mathrm{F}^{\alpha}\right) \in \mathscr{A}_{n+2 s}\left(\pi^{-1}\left(U_{\alpha}\right), V\right)
$$

For that reason, we will denote this generated form by $\left(\chi_{n}^{s} v\right) \bullet \mathrm{F}$ or simply $\chi v \bullet \mathrm{F}$.

Finally we need to compute the exterior derivative of these generated differential forms. We thus cite the following theorem from Ref. 8: 
Theorem III.3: Let $\Gamma$ be a connection on a principal fiber bundle $P(M, G)$ and let $B(M, F, G)$ be an associated bundle, $V$ any vector space, $\chi_{n}^{s} \in \mathscr{f}_{n}(F) \otimes \operatorname{Hom}\left(\otimes{ }^{s} \mathfrak{g}, V\right)$ be $G$-equivariant and $\phi_{n} \in \mathscr{C}_{n}(F) \otimes V$ be invariant under $G$. Then

$$
\begin{array}{r}
d\left(\chi_{n}^{s} v \bullet \mathrm{F}\right)=\left[\left(d \chi_{n}^{s}\right) v\right]_{n+1}^{s} \bullet \mathrm{F}+\left[\left(L \bullet \chi_{n}^{s}\right) v\right]_{n-1}^{s+1} \bullet \mathrm{F}, \\
=\left[\left(d \chi_{n}^{s}\right) v\right]_{n+1}^{s} \bullet \mathrm{F}+\left[\left(L_{\bullet} \chi_{n}^{s}\right) v\right]_{n-1}^{s+1} \bullet \mathrm{F}, \\
d\left(\phi_{n} v\right)=\left(d \phi_{n}\right) v+\left[\left(L_{\bullet} \phi_{n}\right) v\right]_{n-1}^{1} \bullet \mathrm{F} .
\end{array}
$$

Note that the second identity follows from the already mentioned fact that, in combination with F, only the symmetric part of $\left(L_{\bullet} \chi_{n}^{s}\right) v$ counts, cf. Lemma II.2. The last identity is a simple corollary to the first one, since for $s=0, G$-equivariance means invariance, $\operatorname{Hom}\left(\otimes^{0} \mathfrak{g}, V\right)=V$, and $\chi_{r}^{0} \bullet \mathrm{F}=\chi_{r}^{0}$ by definition.

Theorem III.3 proves that the diagram in Section I does not commute in general. Only for 0 -forms $\phi$, i.e., functions $\phi \in C^{\infty}(F, V)$, we know that $L_{\bullet} \phi=0$.

\section{IV. Г-ADAPTED AND G-TRANSGRESSIVE DIFFERENTIAL FORMS}

Now we are prepared for the notion of $\Gamma$-adapted differential forms on a bundle:

Definition IV.1: Let $\Gamma$ be a connection on $P(M, G)$ and $B=P \times{ }_{G} F$. A differential form $\phi^{A}$ $\in \mathscr{A}(B, V)$ is called $\Gamma$-adapted if $\chi^{i} \in \mathscr{C}_{n(i)}\left(F, \operatorname{Hom}\left(\otimes^{s(i)} \mathfrak{g}, V\right)\right)_{\text {equiv }}$ are given such that

$$
\phi^{A}=\sum_{i} \chi^{i} v \cdot \mathrm{F}
$$

It is this splitting into a sum of $\chi^{i} v \bullet \mathrm{F}$ that we have in mind when we say that a form can be presented in such a way that all horizontal terms are given by the gauge fields $\mathrm{F}^{\alpha}$.

We will be concerned with the question whether we can find such a $\Gamma$-adapted representative for a cohomology class in $H^{*}(B)$ that is generated by a 0 -transgressive invariant form $\phi$ $\in \mathscr{b}(F)$. For the physical applications in mind, this concentration on invariant forms is no real restriction. Recall from the general theory of fiber bundles that, for every bundle over a paracompact manifold $M$ with a connected structure group $G$, this Lie group $G$ is reducible to its maximal compact connected subgroup $K$, i.e., $G$ may be a priori chosen to be compact. For example, if we are dealing with electromagnetic interactions, we have $G=\mathrm{U}_{1}$; if we are dealing with electroweak interactions within the Glashow, Salam and Weinberg theory, then $G=\mathrm{SU}_{2} \times \mathrm{U}_{1}$; and for strong interactions covered by quantum chromodynamics $(\mathrm{QCD}), G=\mathrm{SU}_{3}$. In general, for Yang-Mills theories we have $G=\mathrm{U}_{n} \times \mathrm{U}_{n}$ or a subgroup $H<G$.

For any compact Lie group we have the normalized Haar measure $\mu$, and we can project any form $\phi \in \mathscr{C}(F, V)$ onto an invariant form $\phi_{\text {inv }}$ defined by

$$
\phi_{\mathrm{inv}}:=\int_{G} L_{g}^{\star} \phi d \mu(g) .
$$

(Analogous projections onto equivariant forms also exist.) On the other hand, if $G$ is connected, then all maps $L_{g}$ are homotopic to the identity map $L_{e}=\mathrm{id}_{F}$, which yields that $\left[L_{g}^{\star}\right]=\mathrm{id}_{H^{*}(F)}$. Thus, if we denote the cohomology of the invariant closed forms on $F$ modulo the invariant exact forms by $H_{\text {inv }}^{*}(F)$, we have the following proposition:

Proposition IV.2: If $G$ is a compact connected Lie group acting on $F$, then $H^{*}(F) \cong H_{\mathrm{inv}}^{*}(F)$, and the isomorphims are induced by the above projection onto invariant forms, resp., the injection $i: \mathscr{A}(F)_{\mathrm{inv}} \rightarrow \mathscr{A}(F)$. 
$\mathscr{A}(F, V)_{\text {inv }}$ contains an important subset, whose elements we will call $G$-transgressive forms. Let $[r]:=\max _{z \in \mathbb{Z}}\{z \leqslant r\}$ for all $r \in \mathbb{R}$. Then their definition is as follows:

Definition IV.3: Let $L: G \times F \rightarrow F$ be a left Lie group action. An invariant closed differential form $\phi_{n} \in \mathscr{b}_{n}(F, V)_{\text {inv }}$ will be called $G$-transgressive if equivariant differential forms $\chi^{i}$ $\in \mathscr{C}_{n-2 i}\left(F, \operatorname{Sym}_{i}(\mathfrak{g}, V)\right)_{\text {equiv }}$ exist for $0 \leqslant i \leqslant[n / 2]$ with

$$
\chi^{0}=\phi_{n},-L_{\bullet}^{\vee} \chi^{i}=d \chi^{i+1} \text { for all } 0 \leqslant i \leqslant[n / 2]-1 \text { and } L_{\bullet}^{\vee} \chi^{[n / 2]}=0
$$

Denote the set of all $G$-transgressive forms on $F$ by $\mathscr{A}(F, V)_{G-\text { trans }}$.

Note IV.4: The reader who is familiar with equivariant cohomology will recognize that $G$-transgressive forms define equivariant cohomology classes for the given $G$-manifold $F$, cf. Atiyah and Bott ${ }^{9}$ or Mathai and Quillen ${ }^{10}$ and Section VIII.

Recall the definition of the exterior product of vector valued differential forms from (1). For $K_{r} \in \operatorname{Sym}_{r}(\mathfrak{g}, \mathbb{R})$ and $K_{s} \in \operatorname{Sym}_{s}(\mathfrak{g}, \mathbb{R})$ we have the symmetric product $K_{r} \vee K_{s}:=\operatorname{Sym}\left(K_{r} \otimes K_{s}\right)$. For any bilinear $\varphi: V \times V \rightarrow V$ this extends to a bilinear mapping on the (infinite dimensional) vector $\operatorname{space} \operatorname{Sym}(\mathfrak{g}, V):=\oplus_{s=0}^{\infty} \operatorname{Sym}_{s}(\mathfrak{g}, V)$ analogously to $(1)$, which we also denote by $\vee$. This in turn defines the exterior product $\wedge_{\vee}$. With respect to $\wedge_{V}$, the set $\mathscr{C}(F) \otimes \operatorname{Sym}(\mathfrak{g}, V)$ is an exterior algebra with subalgebra $\mathscr{C}(F)_{\text {equiv }} \otimes \operatorname{Sym}(\mathfrak{g}, V)$.

Lemma IV.5: $d$ and $L_{\bullet}^{\vee}$ are skew-derivations of degree 1, resp. - 1, of the algebras $\mathscr{A}(P) \otimes \operatorname{Sym}(\mathfrak{g}, V)$ and $\mathscr{A}(P)_{\text {equiv }} \otimes \operatorname{Sym}(\mathfrak{g}, V)$. For all $\alpha_{n} \in \mathscr{C}_{n}(P) \otimes \operatorname{Sym}(\mathfrak{g}, V)$ and $\omega$ $\in A(P) \otimes \operatorname{Sym}(\mathfrak{g}, V)$,

$$
\begin{gathered}
d\left(\alpha_{n} \wedge_{\bigvee} \omega\right)=\left(d \alpha_{n}\right) \wedge_{\bigvee} \omega+(-1)^{n} \alpha_{n} \wedge_{\bigvee}(d \omega), \\
L_{\bullet}^{\vee}\left(\alpha_{n} \wedge_{\bigvee} \omega\right)=\left(L_{\bullet}^{\vee} \alpha_{n}\right) \wedge_{\bigvee} \omega+(-1)^{n} \alpha_{n} \wedge_{\bigvee}\left(L \vee V_{\bullet} \omega\right) .
\end{gathered}
$$

Lemma IV.5 is the main ingredient in the proof of the following proposition:

Proposition IV.6: $\mathscr{b}(F, V)_{G-\text { trans }}$ is a R-subalgebra of $\mathscr{C}(F, V)$, whenever a bilinear map $\varphi: V \times V \rightarrow V$ and thus a wedge product $\wedge_{\varphi}$ is defined. If $\phi_{m}$ and $\psi_{n}$ are $G$-transgressive and $\chi^{i} \in \mathscr{A}_{m-2 i}\left(F, \operatorname{Sym}_{i}(\mathfrak{g}, V)\right)_{\text {equiv }}$, resp. $\xi^{i} \in \mathscr{A}_{n-2 j}\left(F, \operatorname{Sym}_{j}(\mathfrak{g}, V)\right)_{\text {equiv }}$, are the differential forms given by (10) for $\phi_{m}$, resp. $\psi_{n}$, then

$$
\zeta^{k}:=\sum_{i+j=k} \chi^{i} \wedge_{\vee} \xi^{j} \in \mathscr{A}_{m+n-2 k}\left(F, \operatorname{Sym}_{k}(\mathfrak{g}, V)\right)_{\mathrm{equiv}}
$$

for $0 \leqslant k \leqslant[m / 2]+[n / 2]$ (and $\zeta^{[(m+n) / 2]}:=0$ if $m$ and $n$ are odd) are the corresponding forms for $\phi_{m} \wedge \psi_{n}$.

Proof: Obviously $\mathscr{A}(F, V)_{G-\text { trans }}$ is a R-subspace of $\mathscr{A}(F, V)$. Hence we only have to check if the $\zeta^{k}$, for $0 \leqslant k \leqslant[(m+n) / 2]$, obey the identities (10) for $\phi_{m} \wedge_{\vee} \psi_{n}=\phi_{m} \wedge_{\varphi} \psi_{n}$ $\in A_{m+n}(F, V)_{\text {inv }}$. Obviously $\phi_{m} \wedge{ }_{\vee} \psi_{n}$ is closed and $\zeta^{0}=\chi^{0} \wedge{ }^{0} \xi^{0}=\phi_{m} \wedge{ }_{\vee} \psi_{n}$. By definition of $\wedge_{\vee}$, the differential forms $\zeta^{k}$ are elements of $\mathscr{C}_{m+n-2 k}\left(F, \operatorname{Sym}_{k}(\mathfrak{g}, V)\right)$ and

$$
L_{g}^{\star} \zeta^{k}=\sum_{i+j=k} L_{g}^{\star} \chi^{i} \wedge_{\bigvee} L_{g}^{\star} \xi^{j}=\sum_{i+j=k}\left(\operatorname{Ad}\left(g^{-1}\right)^{\star}\right)_{\star} \chi^{i} \wedge_{\bigvee}\left(\operatorname{Ad}\left(g^{-1}\right)^{\star}\right)_{\star} \xi^{j}=\left(\operatorname{Ad}\left(g^{-1}\right)^{\star}\right)_{\star} \zeta^{k}
$$

whence all $\zeta^{k}$ are $G$-equivariant. Next for $0 \leqslant k \leqslant[m / 2]+[n / 2]-1$, we obtain from Lemma IV.5, using $d \chi^{0}=d \xi^{0}=0$, 


$$
\begin{aligned}
d \xi^{k+1}= & \sum_{i+j=k+1} d\left(\chi^{i} \wedge_{\bigvee} \xi^{j}\right) \\
= & \sum_{i+j=k+1}\left[d \chi^{i} \bigwedge_{\bigvee} \xi^{j}+(-1)^{m-2 i} \chi^{i} \bigwedge_{\bigvee} d \xi^{j}\right] \\
= & d \chi^{0} \bigwedge_{\bigvee} \xi^{k+1}+\sum_{i+j=k}\left[d \chi^{i+1} \bigwedge_{\bigvee} \xi^{j}+(-1)^{m} \chi^{i} \bigwedge_{\bigvee} d \xi^{j+1}\right] \\
& +(-1)^{m} \chi^{k+1} \bigwedge_{\bigvee} d \xi^{0} \\
= & -\sum_{i+j=k}\left[L_{\bullet} \chi^{i} \bigwedge_{\bigvee} \xi^{j}+(-1)^{m} \chi^{i} \bigwedge_{\bigvee} L_{\bullet} \xi^{j}\right] \\
= & \left.-\sum_{i+j=k} L_{\bullet} \bullet \chi^{i} \bigwedge_{\bigvee} \xi^{j}\right)=-L_{\bullet} \xi^{k} .
\end{aligned}
$$

Analogously, since $L_{\bullet}^{\vee} \chi^{[m / 2]}=L_{\bullet}^{\vee} \xi^{[n / 2]}=0$,

$$
\begin{aligned}
L_{\bullet}^{\vee} \zeta^{[m / 2]+[n / 2]} & =\sum_{i+j=[m / 2]+[n / 2]} L_{\bullet}^{\bigvee}\left(\chi^{i} \bigwedge_{\bigvee} \xi^{j}\right) \\
& =L_{\bullet} \chi^{[m / 2]} \bigwedge_{\bigvee} \xi^{[n / 2]}+(-1)^{m} \chi^{[m / 2]} \bigwedge_{\bigvee} L_{\bullet} \xi^{[n / 2]}=0 .
\end{aligned}
$$

But $[m / 2]+[n / 2]=[(m+n) / 2]$, except if $m$ and $n$ are both odd, where $[m / 2]+[n / 2]=[(m+n) / 2]-1$. In that case, we have just shown that $-L_{\bullet} \zeta^{[(m+n) / 2]-1}=0$, whence we may choose $\zeta^{[(m+n) / 2]}:=0$. This completes the proof that $\phi_{m} \wedge_{\vee} \psi_{n}$ is $G$-transgressive.

Now we are ready for the following theorem:

Theorem IV.7: Let $\Gamma$ be a connection on a principal bundle $P(M, G)$ and $B=P \times{ }_{G} F$ an associated bundle with left Lie group action $L: G \times F \rightarrow F$. Let $V$ denote any vector space. If the form $\phi_{n} \in \mathscr{A}_{n}(F, V)_{\mathrm{inv}}$ is $G$-transgressive and the equivariant forms $\chi_{n-2 i}^{i}$ $\in \mathscr{A}_{n-2 i}\left(F, \operatorname{Sym}_{i}(\mathfrak{g}, V)\right)_{\text {equiv }}$ are given by (10), then

$$
\phi_{n}^{A}:=\sum_{i=0}^{[n / 2]}\left(\chi_{n-2 i}^{i} v\right) \cdot \mathrm{F} \in \mathscr{C}_{n}(B, V)
$$

is closed and $\Gamma$-adapted. Its restriction to the fibers is $\phi_{n}$, i.e., for any $\alpha \in A$ and all $x \in U_{\alpha}$, we have $i_{\alpha, x}^{\star} \phi_{n}^{A}=\phi_{n}$.

Proof: $\phi_{n}^{A}$ is obviously adapted to $\Gamma$. Furthermore Theorem III.3 yields

$$
\begin{aligned}
d \phi_{n}^{A} & =\sum_{i=0}^{[n / 2]}\left(d \chi_{n-2 i}^{i}\right) v \cdot \mathrm{F}+\left(L \bullet \chi_{n-2 i}^{i}\right) v \cdot \mathrm{F} \\
& =\left(d \phi_{n}\right) v+\sum_{i=0}^{[n / 2]-1}\left(d \chi_{n-2 i-2}^{i+1}+L \bullet \chi_{n-2 i}^{i}\right) v \cdot \mathrm{F}+\left(L_{\bullet}^{\vee} \chi_{n-2[n / 2]}^{[n / 2]}\right) v \cdot \mathrm{F}=0,
\end{aligned}
$$

since $\phi_{n}$ is $G$-transgressive. Finally, since $i_{\alpha, x}^{\star} \pi^{\star} \mathrm{F}^{\alpha}=0$ for all $x \in U_{\alpha}$, we obtain $i_{\alpha, x}^{\star} \phi_{n}^{A}=i_{\alpha, x}^{\star}\left(\chi^{0} v\right)=i_{\alpha, x}^{\star}\left(\phi_{n} v\right)$. But naturally $i_{\alpha, x}^{\star}\left(\phi_{n} v\right)=\phi_{n}$.

Note that the property of being $G$-transgressive only depends on $L$ and $F$. Thus $G$-transgressive forms define de Rham cohomology classes on all fiber bundles where $L$ is the 
action of the structure group $G$ on the fiber $F$. In particular, this condition is independent of the base $M$ and of the question whether the bundle is trivial or not. Indeed we have the following:

Corollary IV.8: Let $L: G \times F \rightarrow F$ be a left Lie group action. If a form $\phi_{n} \in \mathscr{A}_{n}(F)$ is $G$-transgressive, it is 0 -transgressive for any bundle $B(M, F, G)$ that comes along with $L$. Thus $\phi_{n}$ defines a unique cohomology class $\left[\phi_{n}^{A}\right] \in H^{n}(B)$ with $\left[i_{\alpha, x}^{\star}\right]\left[\phi_{n}^{A}\right]=\left[\phi_{n}\right] \in H^{n}(F)$, independently of the paracompact base $M$ and the transition functions $g_{\alpha \beta}$.

Proof: By the existence theorem for connections, every principal bundle $P(M, G)$ over a paracompact manifold $M$ admits a connection $\Gamma$ (Ref. 5, p. 67). Thus $\phi_{n}^{A}$ is well-defined and Theorem IV applies.

Corollary IV.9: If $\Gamma$ and $\Gamma^{\prime}$ are two connections on $P(M, G)$ and $\phi \in \mathscr{A}(F)$ is $G$-transgressive, then there exists $\psi \in \mathscr{C}(B)$ such that the forms $\phi^{A}$ and $\phi^{A^{\prime}}$ obey:

$$
\phi^{A}-\phi^{A^{\prime}}=d \psi \quad \text { with } d\left(i_{\alpha, x}^{\star} \psi\right)=0
$$

Let us derive the analogue to Theorem IV.7 for 1-dimensional Abelian Lie groups $G$ like the electromagnetic structure group $G_{\mathrm{em}} \cong \mathrm{U}_{1} \cong \mathrm{S}^{1}$. According to the following lemma, we may reformulate the notion of $G$-transgressive forms in that case:

Lemma IV.10: If $G$ is Abelian with $\mathfrak{g}=E \mathbb{R}$, then $\phi_{n} \in \mathscr{A}(F, V)_{\text {inv }}$ is $G$-transgressive iff $\chi^{i}$ $\in \mathscr{C}_{n-2 i}(F, V)_{\mathrm{inv}}$ exist for $0 \leqslant i \leqslant[n / 2]$ such that with $\nu^{i}:=\iota_{\mathscr{C}_{E}} \chi^{i}$ the following equations hold:

$$
\chi^{0}=\phi_{n},-\nu^{i}=d \chi^{i+1} \text { for all } 0 \leqslant i \leqslant[n / 2]-1 \text { and } \nu^{[n / 2]}=0 .
$$

Also since $\mathfrak{g} \cong \mathrm{R}$, we can replace $\bullet$ by the exterior product and as a corollary to Theorem IV.7 and Lemma II.2 we obtain the following.

Theorem IV.11: Let $\Gamma$ be a connection on a principal bundle $P(M, G)$, where $G$ is Abelian with $\mathfrak{g}=E \mathbb{R}$, and let $B=P \times{ }_{G} F$ be any associated bundle with left Lie group action $L: G \times F \rightarrow F$. If $\phi_{n} \in \mathscr{A}_{n}(F, V)_{\mathrm{inv}}$ is $G$-transgressive and $\chi_{n-2 i}^{i} \in \mathscr{A}_{n-2 i}(F, V)_{\mathrm{inv}}$ are given by (11), then with $\widetilde{\mathrm{F}}:=1 / E \pi^{\star} \mathrm{F} \in \mathscr{A}_{2}(B)$,

$$
\begin{aligned}
& \phi_{n}^{A}=\sum_{i=0}^{[n / 2]}\left(\chi_{n-2 i}^{i} v\right) \underbrace{\tilde{\mathrm{F}} \wedge \ldots \wedge \widetilde{\mathrm{F}}}_{i} \\
& =\sum_{i=0}^{[n / 2]} \underbrace{\widetilde{\mathrm{F}}-\wedge \ldots \wedge \widetilde{\mathrm{F}}}_{i} \wedge\left(\chi_{n-2 i}^{i} v\right) \in \mathscr{\ell}_{n}(B, V)
\end{aligned}
$$

is closed and $\Gamma$-adapted. Its restriction to the fibers is $\phi_{n}$, i.e., for any $x \in U_{\alpha}, i_{\alpha, x}^{\star} \phi_{n}^{A}=\phi_{n}$.

Finally, in order to evaluate Theorem IV.11, we note that for any form $\phi \in \mathscr{A}(F, V)$, the vertical projections (8) are given by

$$
\left.\phi v\right|_{\pi^{-1}\left(U_{\alpha}\right)}=\left(\pi_{\alpha}^{\star} \phi\right) v^{\alpha}=\pi_{\alpha}^{\star} \phi+\widetilde{\mathrm{A}}^{\alpha} \wedge \pi_{\alpha}^{\star}\left(l_{\mathscr{L}_{E}} \phi\right)
$$

with $\widetilde{\mathrm{A}}^{\alpha}:=1 / E \pi^{\star} \mathrm{A}^{\alpha}{ }^{7}$ Hence, since $\left(l_{\mathscr{C}_{E}}\right)^{2}=0$, we have $\left.\left(l_{\mathscr{C}_{E}} \phi\right) v\right|_{\pi^{-1}\left(U_{\alpha}\right)}=\pi_{\alpha}^{\star}\left(l_{\mathscr{L}_{E}} \phi\right)$.

\section{SKYRMION BUNDLE AND RELATED YANG-MILLS THEORIES}

These results are quite important for the skyrmion bundle in theoretical nuclear physics which treats interactions of mesons and baryons — described within the (ungauged) Skyrme model ${ }^{1,3}$ 
with electromagnetic fields. ${ }^{11-13}$ In order to show this, we need some basic results from current algebra. Denote the left and right invariant 1 -forms on a Lie group $H \leqslant \mathrm{Gl}_{n}(\mathrm{C})$ by $\Theta^{L}$ $\in \mathscr{A}_{1}^{L}(H, \mathfrak{h})$, resp., $\Theta^{R} \in \mathscr{b}_{1}^{R}(H, \mathfrak{h})$, i.e.,

$$
\Theta_{h}^{L}(h \cdot X)=\Theta_{h}^{R}(X \cdot h)=X \text { for all } h \in H, X \in \mathfrak{h},
$$

where $h \cdot X$ and $X \cdot h$ are elements of $T_{h}(H)$. In the literature, these forms are also called invariant "currents" and we find the notations $h^{-1} d h$ and $L$ for $\Theta^{L}$, resp., $d h h^{-1}$ and $R$ for $\Theta^{R}$. If $\wedge$ denotes the exterior product of matrix-valued forms with respect to matrix multiplication, then for all $k \in \mathbb{N}$,

$$
\left(\Theta^{L}\right)^{k}:=\underbrace{\Theta^{L} \wedge \ldots \wedge \Theta^{L}}_{k} \in \mathcal{A}_{k}^{L}\left(H, \mathbb{C}^{n \times n}\right)
$$

and

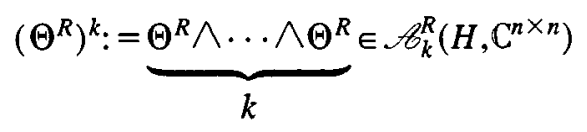

are well-defined left, resp., right, invariant $k$-forms on $H$, and so are

$$
\lambda_{k}^{M}:=\operatorname{Tr}\left[M \cdot\left(\Theta^{L}\right)^{k}\right] \in \mathscr{C}_{k}^{L}(H, \mathrm{C})
$$

and

$$
\rho_{k}^{M}:=\operatorname{Tr}\left[M \cdot\left(\Theta^{R}\right)^{k}\right] \in \mathscr{C}_{k}^{R}(H, \mathrm{C})
$$

for any matrix $M \in \mathrm{C}^{n \times n}$. Especially for $M=1$, we obtain the bi-invariant

$$
\omega_{k}:=\lambda_{k}^{1}=\rho_{k}^{1} \in \mathscr{C}_{k}(H, \mathrm{C})_{\mathrm{inv}},
$$

and one easily checks that $\omega_{2 k}=0$. Now the Maurer-Cartan identities yield the following.

Lemma V.1: $d\left(\Theta^{L}\right)^{2 k-1}=-\left(\Theta^{L}\right)^{2 k}$ and $d\left(\Theta^{R}\right)^{2 k-1}=\left(\Theta^{R}\right)^{2 k}$. As a consequence for any matrix $M \in \mathrm{C}^{n \times n}$, we have $d \lambda_{2 k-1}^{M}=-\lambda_{2 k}^{M}, d \rho_{2 k-1}^{M}=\rho_{2 k}^{M}$ hence $d \omega_{2 k-1}=0$ and $d \lambda_{2 k}^{M}=d \rho_{2 k}^{M}=0$.

In addition, recall that the cohomology of $\mathrm{SU}_{n}$ and $\mathrm{U}_{n}$ is generated as an algebra by $\omega_{3}$, $\omega_{5}, \ldots, \omega_{2 n-1}$ for $\mathrm{SU}_{n}$, resp., by $\omega_{1}, \omega_{3}, \ldots, \omega_{2 n-1}$ for $\mathrm{U}_{n}$, cf. Greub, Halperin, and Vanstone. ${ }^{14}$

In the ungauged Skyrme model, the meson fields occur as maps $U: M \rightarrow \mathrm{SU}_{n}$, where $M$ denotes space-time and $n$ denotes the number of flavors in $Q C D$. The configuration $U \equiv \rrbracket$ represents the vacuum. Baryons appear as topological soliton solutions, as "skyrmions," of these fields. The number of baryons represented by a given mesonic field configuration is computed by an integration of $U^{\star} \omega_{3}$ over the space manifold (which is compactified at infinity, where the fields are required to tend to the vacuum value 1 ). For $n \geqslant 3$, the action integral splits into two parts, the nonamomalous action and the Wess-Zumino term. The latter is an integral over the differential form $\omega_{5}{ }^{2}$ 
In order to treat interactions with electromagnetic fields (especially those of magnetic monopoles, $\left.{ }^{11,12}\right)$ one constructs a fiber bundle $B\left(M, \mathrm{SU}_{n}, G_{\mathrm{em}}\right)$, cf. Section I. If $e$ denotes the electric unit charge, then the left action of $G_{\mathrm{em}}=1 / e \cdot \mathrm{S}^{1}$ on $\mathrm{SU}_{n}$ is given by the inner automorphisms

$$
L(g, U):=e^{-i e g Q} U e^{+i e g Q}
$$

for $g \in G_{\mathrm{em}}$ and $U \in \mathrm{SU}_{n} \cdot Q$ is the $n \times n$-matrix containing the quark charges in units of $e$ : for $n=2,3$,

$$
Q=\left(\begin{array}{cc}
\frac{2}{3} & 0 \\
0 & -\frac{1}{3}
\end{array}\right), \text { resp., } Q=\left(\begin{array}{ccc}
\frac{2}{3} & 0 & 0 \\
0 & -\frac{1}{3} & 0 \\
0 & 0 & -\frac{1}{3}
\end{array}\right)
$$

We put $\phi^{\alpha}:=\pi_{\alpha}^{\star} \phi$ for any $\phi \in \mathscr{A}(F, V)$. Under a change of bundle charts we then have

$$
U^{\alpha}(x)=L\left(g_{\alpha \beta}, U^{\beta}\right)(x)=e^{-i e g_{\alpha \beta}(x) Q} U^{\beta}(x) e^{+i e g_{\alpha \beta}(x) Q},
$$

and for the canonical vector field $\mathscr{L}_{E} \in \mathscr{D}^{1}\left(\mathrm{SU}_{n}\right)$ induced by $E=1 / e \in \mathfrak{g}$, we obtain

$$
\mathscr{L}_{E}(U)=-i[Q, U] \text { for all } U \in \mathrm{SU}_{n} \text {. }
$$

Now (12) yields

$$
\left(d U^{\alpha}\right) v=d U^{\alpha}-i e \mathrm{~A}^{\alpha}\left[Q, U^{\alpha}\right]
$$

For the invariant forms $\rho_{k}^{Q}, \lambda_{k}^{Q}$ and $\omega_{2 k+1}$, we obtain the following lemma: ${ }^{13}$

Lemma V.2: For the action given in (13),

$$
\begin{gathered}
\boldsymbol{l}_{\mathscr{L}_{E}} \omega_{2 k+1}=-(2 k+1) i\left(\rho_{2 k}^{Q}-\lambda_{2 k}^{Q}\right), \\
\boldsymbol{l}_{\mathscr{L}_{E}}\left(\rho_{2 k}^{Q}-\lambda_{2 k}^{Q}\right)=0, \\
\boldsymbol{l}_{\mathscr{L}_{E}}\left(\rho_{2 k+1}^{Q}+\lambda_{2 k+1}^{Q}\right)=-2 i \sum_{j=1}^{k} \operatorname{Tr}\left[Q U\left(\Theta^{L}\right)^{2 j-1} Q\left(\Theta^{L}\right)^{2 k-2 j+1} U^{-1}\right] \\
-i \sum_{j=0}^{k} \operatorname{Tr}\left[Q\left(\Theta^{R}\right)^{2 j} Q\left(\Theta^{R}\right)^{2 k-2 j}-Q\left(\Theta^{L}\right)^{2 j} Q\left(\Theta^{L}\right)^{2 k-2 j}\right] .
\end{gathered}
$$

In order to define a baryon number and an anomalous action for the skyrmion bundle, we have to extend the forms $\omega_{3}$ and $\omega_{5}$ to the bundle. Several approaches "by trial and error" have been made to "generalize"' $\omega_{3}$ and $\omega_{5}$, cf. Callan and Witten, ${ }^{11}$ Kaymakcalan et al., ${ }^{15}$ or Pak and Rossi. $^{16}$ In terms of the language we are using, we would like to obtain differential forms $\omega_{3}^{\mathrm{A}}$ and $\omega_{5}^{\mathrm{A}}$ that are adapted to the Maxwell connection given by the electromagnetic fields. Thus we will examine whether $\omega_{3}$ and $\omega_{5}$ are $G_{\mathrm{em}}$-transgressive.

This is indeed the case. According to Lemma IV.10 we have to find $\chi_{n-2 i}^{i} \in \mathscr{C}_{n}\left(\mathrm{SU}_{n}, \mathrm{C}\right)$ and $\nu_{n-2 i-1}^{i}=l_{\mathscr{L}_{E}} \chi_{n-2 i}^{i}$ that obey (11) for $\phi=\omega_{3}$, resp., $\phi=\omega_{5}$. From Lemma V.2 we conclude that for $\phi=\omega_{2 k+1}$, we have $\nu_{2 k}^{0}=-(2 k+1) i\left(\rho_{2 k}^{Q}-\lambda_{2 k}^{Q}\right)$. Now Lemma V.1 yields that 
$\rho_{2 k}^{Q}-\lambda_{2 k}^{Q}=d\left(\rho_{2 k-1}^{Q}+\lambda_{2 k-1}^{Q}\right)$, so $\chi_{2 n-1}^{1}=(2 k+1) i\left(\rho_{2 k-1}^{Q}+\lambda_{2 k-1}^{Q}\right)$. For $\omega_{3}$ we are already done, since $\chi_{1}^{1}$ is global and vertical due to Lemma V.2: $\nu_{0}^{1}=0$. For $\chi_{3}^{1}$, again Lemma V.2 yields $\nu_{2}^{2}=-10 i^{2} \operatorname{Tr}\left[Q^{2}\left(\left(\Theta^{R}\right)^{2}-\left(\Theta^{L}\right)^{2}\right)+Q d U^{-1} \wedge Q d U\right]$. One easily verifies that

$$
\chi_{1}^{2}=10 i^{2}\left(\rho_{1}^{Q^{2}}+\lambda_{1}^{Q^{2}}\right)+5 i^{2} \operatorname{Tr}\left(Q d U Q U^{-1}-Q U Q d U^{-1}\right)+r i^{2} d \operatorname{Tr}\left(Q U^{-1} Q U\right), r \in \mathbb{R},
$$

is an admissible choice and that $\nu_{0}^{2}=0$, thus $\chi_{1}^{2}$ is global and vertical. For physical reasons (parity invariance ${ }^{15}$ ), we put $r=0$. We thus obtain from Theorem IV.11: ${ }^{13}$ the following.

Theorem V.3: $\omega_{3}$ and $\omega_{5}$ are $G_{\mathrm{em}}$-transgressive and generate de Rham cohomology groups isomorphic to $\mathbb{R}$ for any skyrmion bundle. Representatives for the generated cohomology groups, that are adapted to the Maxwell connection, are

$$
\begin{gathered}
\omega_{3}^{\mathrm{A}}=\omega_{3} v+i e \mathrm{~F} \wedge \chi_{1}^{1} v \\
=\left[\omega_{3}^{\alpha}-3 i e \mathrm{~A}^{\alpha} \wedge\left(\rho_{2}^{Q}-\lambda_{2}^{Q}\right)\right]+3 i e \mathrm{~F} \wedge\left(\rho_{1}^{Q}+\lambda_{1}^{Q}\right), \\
\omega_{5}^{\mathrm{A}=} \omega_{5} v+i e \mathrm{~F} \wedge \chi_{3}^{1} v+(i e)^{2} \mathrm{~F} \wedge \mathrm{F} \wedge \chi_{1}^{2} v \\
=\left[\omega_{5}^{\alpha}-5 i e \mathrm{~A}^{\alpha} \wedge\left(\rho_{4}^{Q}-\lambda_{4}^{Q}\right)\right]+5 i e \mathrm{~F} \wedge\left\{\left(\rho_{3}^{Q}+\lambda_{3}^{Q}\right)^{\alpha}\right. \\
\left.-2 i e \mathrm{~A}^{\alpha} \wedge \operatorname{Tr}\left[Q^{2}\left(\left(\Theta^{R}\right)^{2}-\left(\Theta^{L}\right)^{2}\right)+Q d U^{-1} \wedge Q d U\right]^{\alpha}\right\}+5(i e)^{2} \mathrm{~F} \wedge \mathrm{F} \wedge\left[2\left(\rho_{1}^{Q^{2}}+\lambda_{1}^{Q^{2}}\right)^{\alpha}\right. \\
\left.+\operatorname{Tr}\left(Q d U Q U^{-1}-Q U Q d U^{-1}\right)^{\alpha}\right] .
\end{gathered}
$$

In fact, one can prove that all differential forms $\omega_{2 k-1}$ are $G_{\text {em }}$-transgressive. ${ }^{17}$

In comparison to the literature cited, our formalism has led to quite compact notations for $\omega_{3}^{\mathrm{A}}$ and $\omega_{5}^{\mathrm{A}}$. This advantage becomes even more obvious when generalizations to other gauge groups, especially non-Abelian gauge groups, are considered, e.g., instead of $G \cong \mathrm{S}^{1}$ and $F=\mathrm{SU}_{n}$ take a Yang-Mills theory where $G=\mathrm{U}_{n}^{L} \times \mathrm{U}_{n}^{R}$ and $F=\mathrm{U}_{n}$ with $L_{\left(g_{L}, g_{R}\right)}(U)=g_{L} U g_{R}^{-1}$. As a generalization of (14) we have for all $\left(X_{L}, X_{R}\right) \in \mathrm{u}_{n}^{L} \oplus \mathrm{u}_{n}^{R}$

$$
\mathscr{L}_{\left(X_{L}, X_{R}\right)}(U)=X_{L} U-U X_{R} \text { for all } U \in \mathrm{SU}_{n} .
$$

Now the gauge fields take their values in $\mathrm{u}_{n}^{L} \oplus \mathrm{u}_{n}^{R}$, i.e., $\mathrm{A}^{\alpha}=\left(\mathrm{A}_{L}^{\alpha}, \mathrm{A}_{R}^{\alpha}\right)$ and $\mathrm{F}^{\alpha}=\left(\mathrm{F}_{L}^{\alpha}, \mathrm{F}_{R}^{\alpha}\right)$ $\in \mathscr{b}\left(U_{\alpha}, \mathrm{u}_{n}^{L} \oplus \mathrm{u}_{n}^{R}\right)$ define the connection $\Gamma$ on $P(M, G)$. In this case, omitting the superscripts $\alpha$, we obtain $d U v=d U+\mathrm{A}_{L} U-U \mathrm{~A}_{R}$, hence

$$
\begin{gathered}
\Theta^{L} v=\Theta^{L}+U^{-1} \mathrm{~A}_{L} U-\mathrm{A}_{R}, \\
\Theta^{R} v=\Theta^{R}+\mathrm{A}_{L}-U \mathrm{~A}_{R} U^{-1}, \\
\omega_{1} v=\omega_{1}+\operatorname{Tr}\left(\mathrm{A}_{L}-\mathrm{A}_{R}\right) .
\end{gathered}
$$

Moreover, we have $L_{\bullet} \omega_{1}=\operatorname{Tr}\left(\pi_{L}-\pi_{R}\right)$ with the projections $\pi^{L / R}: \mathfrak{g}=\mathrm{u}_{n}^{L} \oplus \mathrm{u}_{n}^{R} \rightarrow \mathrm{u}_{n}^{L / R}$. Thus for any LIE subgroup $H<G$, the closed invariant form $\omega_{1}$ is $H$-transgressive iff $\operatorname{Tr}\left(X_{L}-X_{R}\right)=0$ for all $\left(X_{L}, X_{R}\right) \in \mathfrak{h}$, e.g., we could choose a subgroup of the diagonal $D_{n}=\mathrm{U}_{n}^{L} \times \mathrm{U}_{n}^{L}$ in $G$ such that $g_{L}=g_{R}$ for all $\left(g_{L}, g_{R}\right) \in D_{n}$. (Note that this is the case for the skyrmion bundle.) Or we could choose $H=\mathrm{SU}_{n}^{L} \times \mathrm{SU}_{n}^{R}$, resp., a subgroup of $H$. In Section VII we will prove that in the latter case, $\omega_{1}$ is necessarily $H$-transgressive because $\mathrm{SU}_{n}^{L} \times \mathrm{SU}_{n}^{R}$ is semisimple for $n>2$, cf. Theorem VII.4.

For $\omega_{3}$ we obtain $L_{\bullet} \omega_{3}=3 \operatorname{Tr}\left[\left(\Theta^{R}\right)^{2} \pi^{L}-\left(\Theta^{L}\right)^{2} \pi^{R}\right]$, thus

$$
\chi_{1}^{1}:=-3 \operatorname{Tr}\left(\Theta^{R} \pi^{L}+\Theta^{L} \pi^{R}\right) \in \mathscr{C}_{1}\left(\mathrm{U}_{n}, \operatorname{Hom}(\mathfrak{g}, \mathbb{C})\right)
$$


obeys $d \chi_{1}^{1}=-L_{\bullet} \omega_{3}$ due to Lemma V.1. Omitting the symmetrization $V$, we compute $L \vee \chi_{1}^{1}=3 \operatorname{Tr}\left(\pi^{R} \pi^{R}-\pi^{L} \pi^{L}\right)$, i.e.,

$$
\left(L_{\bullet}^{\vee} \chi_{1}^{1}\right)\left(\left(X_{L}, X_{R}\right),\left(Y_{L}, Y_{R}\right)\right)=3 \operatorname{Tr}\left(X_{R} Y_{R}-X_{L} Y_{L}\right) \neq 0
$$

Thus $\omega_{3}$ is not $G$-transgressive. In fact, take any $\tilde{\chi}_{1}^{1} \in \mathscr{C}_{1}\left(U_{n}, \operatorname{Hom}(\mathfrak{g}, \mathbb{C})\right)_{\text {equiv }}$ with $d \tilde{\chi}_{1}^{1}=-L_{\bullet} \omega_{3}$. Then $\xi_{1}^{1}:=\tilde{\chi}_{1}^{1}-\chi_{1}^{1} \in \mathscr{C}_{1}\left(U_{n}, \operatorname{Hom}(\mathfrak{g}, \mathrm{C})\right)_{\text {equiv }}$ with $d \xi_{1}^{1}=0$. Since $H^{1}\left(\mathrm{SU}_{n}\right)=0$, we find $\xi_{0}^{1} \in C^{\infty}\left(U_{n}, \operatorname{Hom}(\mathfrak{g}, \mathbb{C})\right)$ with $d \xi_{0}^{1}=\xi_{1}^{1}$. In fact, we may choose $\xi_{0}^{1}$ equivariant, because $\mathrm{SU}_{n}$ is compact, analogously to (9). But then for all $X, Y \in \mathfrak{g}$,

$$
\begin{aligned}
L_{\bullet} \xi_{1}^{1}(X, Y) & =\left(\boldsymbol{l}_{\mathscr{L}_{X}} d \xi_{0}^{1}\right)(Y)+\left(\boldsymbol{l}_{\mathscr{L}_{Y}} d \xi_{0}^{1}\right)(X) \\
& =\mathscr{L}_{X}\left(\xi_{0}^{1}\right)(Y)+\mathscr{L}_{Y}\left(\xi_{0}^{1}\right)(X) \\
& =\xi_{0}^{1}([Y, X])+\xi_{0}^{1}([X, Y])=0 .
\end{aligned}
$$

Thus $\left(L \vee \widetilde{\chi}_{1}^{1}\right)=\left(L \bigvee \chi_{1}^{1}\right) \neq 0$. Since $\omega_{3}$ is not $G$-transgressive, the generated $\Gamma$-adapted form

$$
\omega_{3}^{\mathrm{A}}=\omega_{3} v+\chi_{1}^{1} v \cdot \mathrm{F} \in \mathscr{C}_{3}\left(B\left(M, \mathrm{U}_{n}, G\right), \mathrm{C}\right)
$$

is not closed in general: $d \omega_{3}^{\mathrm{A}}=\left(L_{\bullet}^{\vee} \chi_{1}^{1}\right) v \cdot \mathrm{F}=\left(L_{\bullet}^{\vee} \chi_{1}^{1}\right) \bullet \mathrm{F}$. Yet if we again restrict $L$ to a subgroup $H<G$ with generators $X^{\sigma}=\left(X_{L}^{\sigma}, X_{R}^{\sigma}\right), \sigma \in I$, such that $\operatorname{Tr}\left(X_{L}^{\sigma} X_{L}^{\tau}\right)=\operatorname{Tr}\left(X_{R}^{\sigma} X_{R}^{\tau}\right)$ for all $\sigma, \tau \in I$, then $L^{\vee} \chi_{1}^{1}=0$ and $\omega_{3}$ is $H$-transgressive. Note that this condition holds for any subgroup of the diagonal $D_{n}$ and thus for the skyrmion bundle.

Finally, some cumbersome calculations show that the voluminous expressions for the WessZumino term in Ref. 15, (4.18), resp., Ref. 2, (24), are equal to the integral over the $\Gamma$-adapted differential form

$$
\omega_{5}^{\mathrm{A}}=\omega_{5} v+\chi_{3}^{1} v \cdot \mathrm{F}+\chi_{1}^{2} v \cdot \mathrm{F} \in \mathscr{C}_{5}\left(B\left(M, \mathrm{U}_{n}, G\right), \mathrm{C}\right),
$$

where the forms $\chi_{5-2 l}^{l} \in \mathscr{A}_{5-2 l}\left(\mathrm{U}_{n}, \operatorname{Sym}_{l}(\mathfrak{g}, \mathrm{C})\right)_{\text {equiv }}$ are given by

$$
\begin{gathered}
\chi_{3}^{1}:=-5 \operatorname{Tr}\left[\left(\Theta^{R}\right)^{3} \pi^{L}+\left(\Theta^{L}\right)^{3} \pi^{R}\right], \\
\chi_{1}^{2}:=10 \operatorname{Tr}\left[\left(\Theta^{R}\right) \pi^{L} \pi^{L}+\left(\Theta^{L}\right) \pi^{R} \pi^{R}\right]+5 \operatorname{Tr}\left(d U \pi^{R} U^{-1} \pi^{L}-d\left(U^{-1}\right) \pi^{L} U \pi^{R}\right) .
\end{gathered}
$$

Analogously to the skyrmion case, one may add a term

$$
r\left[d \operatorname{Tr}\left(\pi^{L} U \pi^{R} U^{-1}\right) v\right] \cdot \mathrm{F}=r d \operatorname{Tr}\left(F_{L} U F_{R} U^{-1}\right), r \in \mathrm{C},
$$

or exclude it by parity invariance. ${ }^{15}$ Also in this case, the differential form $\omega_{5}$ is not $G$-transgressive: we obtain $L_{\bullet} \chi_{1}^{2}=10 \operatorname{Tr}\left(\pi^{L} \pi^{L} \pi^{L}-\pi^{R} \pi^{R} \pi^{R}\right)$, thus again $\omega_{5}$ is $H$-transgressive for any subgroup $H \leqslant D$. More generally, $\omega_{5}$ is $H$-transgressive if and only if the generators of $H$ obey $\operatorname{Tr}\left(X_{L}^{\sigma} X_{L}^{\tau} X_{L}^{v}\right)=\operatorname{Tr}\left(X_{R}^{\sigma} X_{R}^{\tau} X_{R}^{v}\right)$ for all $\sigma, \tau, v \in I$, specifically, only if $\operatorname{Tr}\left[\left(X_{L}^{\sigma}\right)^{3}\right]$ $=\operatorname{Tr}\left[\left(X_{R}^{\sigma}\right)^{3}\right]$ for all $\sigma \in I$, which is the usual condition for cancellation of anomalies on the quark level. $^{2}$

Nevertheless note that $d \omega_{5}^{\mathrm{A}}=\left(L_{\bullet} \chi_{1}^{2}\right) \bullet \mathrm{F}$ consists of a 6 -form on the base. Thus as long as we stick to space-time $M$ - or even a five-dimensional extension - this form vanishes and $\omega_{5}^{\mathrm{A}}$ is in fact closed. The same holds for $\omega_{3}^{\mathrm{A}}$ : although it might not be closed on space-time $M, \omega_{3}^{\mathrm{A}}$ is closed, of course, when restricted to three-dimensional space. 


\section{LIE ALGEBRA COHOMOLOGY}

Back to the general case, we want to derive which closed invariant $n$-forms $\phi_{n}$ on the fiber $F$ are $G$-transgressive in the cases where $n=0,1$ or 2 . To this purpose we need some basic results on Lie algebra cohomology.

Suppose $\mathfrak{g}$ is a $\mathbb{K}$-Lie algebra (for $\mathbb{K}=\mathbb{R}, \mathrm{C}$ ) and $l: \mathfrak{g} \rightarrow \mathrm{gl}(V)$ is a (left) representation of $\mathfrak{g}$ on a (possibly infinte dimensional) $\mathrm{K}$-vector space $V$. Then $\operatorname{Alt}(\mathfrak{g}, V)=\oplus_{p=0}^{\infty} \operatorname{Alt}_{p}(\mathfrak{g}, V)$ becomes a differential complex $C_{l}$ with the following differential operator $\mathbf{d}^{l}=\left(\mathbf{d}_{p}^{l}: C_{l}^{p} \rightarrow C_{l}^{p+1}\right)_{p \in \mathbb{N}_{0}}$ : for $c$ $\in C_{l}^{p}:=\operatorname{Alt}_{p}(\mathfrak{g}, V)$ and $X_{i} \in \mathfrak{g}$,

$$
\begin{aligned}
\mathbf{d}_{p}^{l} c\left(X_{1}, \ldots, X_{p+1}\right):= & \sum_{i=1}^{p+1}(-1)^{i+1} l\left(X_{i}\right)\left(c\left(\ldots, \widehat{X}_{i}, \ldots\right)\right)+\sum_{i=1}^{p} \sum_{j=i+1}^{p+1} \\
& (-1)^{i} c\left(\ldots, \widehat{X}_{i}, \ldots, X_{j-1},\left[X_{i}, X_{j}\right], X_{j+1}, \ldots\right)
\end{aligned}
$$

(where^ indicates that the term is omitted).

Our definition of $\mathbf{d}^{l}$ differs slightly from the definitions in Ref. 14, resp., in Hilgert and Neeb ${ }^{18}$ where analogously to the definition of the exertior derivative $d$, the second term reads

$$
+\sum_{i=1}^{p} \sum_{j=i+1}^{p+1}(-1)^{i+j} c\left(\left[X_{i}, X_{j}\right], X_{1}, \ldots, \widehat{X}_{i}, \ldots, \widehat{X}_{j}, \ldots\right)
$$

Obviously both definitions coincide on $C_{l}$. Nevertheless with our definition not only $\operatorname{Alt}(\mathfrak{g}, V)$ becomes a differential complex, but also $\operatorname{Hom}(\mathscr{T}(\mathfrak{g}), V)$ becomes a complex $\bar{C}_{l}$ with subcomplex $C_{l}$. [ $\mathscr{T}(\mathfrak{g})$ denotes the tensor algebra of $\left.\mathfrak{g}.\right]$ Indeed we can prove — analogously to the proof for $d^{2}=0-$ that $\mathbf{d}_{p+1}^{l} \circ \mathbf{d}_{p}^{l}=0$ on $\bar{C}_{l}$ for any representation $l: \mathfrak{g} \rightarrow \operatorname{gl}(V)$ of $\mathfrak{g}$. Now the cohomology of this complex, $H_{l}^{p}(\mathfrak{g}, V):=H_{\mathbf{d}}^{p}\left(C_{l}\right)$ is called the $p$ th (Chevalley) cohomology space of $\mathfrak{g}$ with values in $V$ with regard to $l$. We put $H_{l}^{p}(\mathfrak{g}):=H_{l}^{p}(\mathfrak{g}, \mathbb{K})$. Analogously, $\bar{H}_{l}^{p}(\mathfrak{g}, V):=H_{\mathbf{d}}^{p}\left(\bar{C}_{l}\right)$ and $\bar{H}_{l}^{p}(\mathfrak{g}):=\bar{H}_{l}^{p}(\mathfrak{g}, \mathbb{K})$.

Lemma VI.1: Let $o: \mathfrak{g} \rightarrow \mathrm{gl}(V)$ denote the trivial representation of $\mathfrak{g}$. Then

(1) $H_{o}^{0}(\mathfrak{g}, V)=\bar{H}_{o}^{0}(\mathfrak{g}, V)=V$.

(2) $H_{o}^{1}(\mathfrak{g}, V)=\bar{H}_{o}^{1}(\mathfrak{g}, V)=\{c \in \operatorname{Hom}(\mathfrak{g}, V) \mid c([\mathfrak{g}, \mathfrak{g}])=\{0\} \leqslant V\}=[\mathfrak{g}, \mathfrak{g}]^{\perp}$, thus $\mathbf{d}_{1}^{o}$ is injective and $H_{o}^{1}(\mathfrak{g}, V)=\{0\}$ for all Lie algebras $\mathfrak{g}$ with $\mathfrak{g}=[\mathfrak{g}, \mathfrak{g}]$, e.g., semisimple Lie algebras.

(3) If $\mathfrak{a}$ is Abelian, then $H_{o}^{p}(\mathfrak{a}, V)=\operatorname{Alt}_{p}(\mathfrak{a}, V)$ and $\bar{H}_{o}^{p}(\mathfrak{a}, V)=\operatorname{Hom}\left(\otimes^{p} \mathfrak{a}, V\right)$.

Moreover, if $V$ is finite dimensional, then Whitehead's lemmas yield that $H_{l}^{1}(\mathfrak{g}, V)=H_{l}^{2}(\mathfrak{g}, V)=0$ for any representation $l: \mathfrak{g} \rightarrow \mathrm{gl}(V)$ of a semisimple Lie algebra $\mathfrak{g}$.

Recall that a double complex $C^{*, *}:=\oplus_{p, q \in \mathrm{N}_{0}} C^{p, q}$ is a doubly graded differential complex with two commuting differential operators, a horizontal operator $\delta: C^{p, q} \rightarrow C^{p+1, q}$ and a vertical operator $d: C^{p, q} \rightarrow C^{p, q+1}$. Every double complex is associated with a singly graded complex $C^{*}$ by summing along the antidiagonal lines, i.e., $C^{n}$ is given by $C^{n}=\oplus_{p+q}{ }_{n} C^{p, q}$. The (total) cohomology of such a double complex is then defined to be the cohomology of the associated singly graded complex with regard to the differential operator $D=\delta+(-1)^{p} d$ on $C^{p, q}$. Note that indeed $D: C^{n} \rightarrow C^{n+1}$. The alternating sign guaranties that $D \circ D=0 .^{6}$

Given a Lie group action $L: G \times F \rightarrow F$, we want to combine the invariant cohomology on $F$ with the Lie algebra cohomology of $\mathfrak{g}$. To this purpose, we form the double complex

$$
\begin{aligned}
C^{*, *}: & =\mathscr{A}(F) \otimes \operatorname{Hom}(\mathscr{T}(\mathfrak{g}), V) \\
& =\oplus_{p, q \in \mathbb{N}_{0}} \mathscr{A}_{q}\left(F, \operatorname{Hom}\left(\otimes^{p} \mathfrak{g}, V\right)\right) .
\end{aligned}
$$


$(-1)^{p} d_{(q)}: C^{p, q} \rightarrow C^{p, q+1}$ is the vertical operator, and for the horizontal operator we have $\delta:=-\mathbf{d}_{(q)}^{l}: C^{p, q} \rightarrow C^{p+1, q}$. For the representation $l: \mathfrak{g} \rightarrow \mathrm{gl}(\mathscr{A}(F, V))$ several choices are possible. e. g., one can take the trivial representation $o$. Then $\mathbf{d}^{o}$ and $d$ obviously commute.

Instead we choose $l$ defined by $l(X):=-L_{\mathscr{L}_{X}}$, where $L_{\mathscr{X}}: \mathscr{A}(F, V) \rightarrow \mathscr{b}(F, V)$ denotes the Lie derivative of forms with respect to a vector field $\mathscr{X} \in \mathscr{D}^{1}(F)$. Recall that $L_{\mathscr{C}}$ is given by $L_{\mathscr{C}}=d \bigcirc_{\mathscr{C}}+\iota_{\mathscr{X}} \bigcirc d$. Since Lie differentiation and exterior differentiation commute, $\delta$ and $d$ commute on the double complex and define an operator $D$.

With regard to these operators we obtain (Ref. 8, Lemma 3.4) the following.

Lemma VI.2: For all $\omega_{n} \in \mathscr{A}_{n}(F, V)$ and all $i \leqslant n+1$,

$$
L_{\bullet}^{i} d \omega_{n}-(-1)^{i} d L_{\bullet}^{i} \omega_{n}=\delta_{i-1} L_{\bullet}^{i-1} \omega_{n} .
$$

$A^{*, *}:=\mathscr{A}(F) \otimes \operatorname{Alt}(\mathfrak{g}, V)$ is a subcomplex of $C^{*, *}$ and $A_{\text {inv }}^{*, *}:=\mathscr{A}(F)_{\mathrm{inv}} \otimes \operatorname{Alt}(\mathfrak{g}, V)$ and $A_{\text {equiv }}^{*, *}:=\mathscr{A}(F)_{\text {equiv }} \otimes \operatorname{Alt}(\mathfrak{g}, V)$ are subcomplexes of $A^{*, *}$, on which the horizontal operators are given by $\delta=-\mathbf{d}^{o}$, resp., $\delta=+\mathbf{d}^{o}$.

Recall that a chain map $f: A \rightarrow B$ between two differential complexes $A$ and $B$ is a homomorphism that commutes with the differential operators of $A$ and $B: f \circ D_{A}=D_{B} \circ f$, e.g., all pullbacks $f^{\star}: \mathscr{b}(M, V) \rightarrow \mathscr{A}(N, V)$ are chain maps.

Definition VI.3: For any Lie group action $L: G \times F \rightarrow F$, the homomorphism $\mathbf{L}: \mathscr{A}(F, V) \rightarrow \mathscr{A}(F) \otimes \operatorname{Alt}(\mathfrak{g}, V)$ is defined by $\mathbf{L} \omega_{n}:=\sum_{i=0}^{n} L_{\bullet}^{i} \omega_{n}$ for all $\omega_{n} \in \mathscr{A}_{n}(F, V)$.

The homomorphism $L_{\bullet}^{*}: \mathscr{A}(F, V) \rightarrow \operatorname{Alt}(\mathfrak{g}, V)$ is given by $L_{\bullet}^{*} \omega:=\sum_{n=0}^{\infty} L_{\bullet}^{n} \omega_{n}$ for all $\omega=\sum_{n=0}^{\infty} \omega_{n}$ with $\omega_{n} \in \mathscr{C}_{n}(F, V)$.

Let $p_{0}: \not{b}(F) \otimes \operatorname{Alt}(\mathfrak{g}, V) \rightarrow \mathscr{b}(P, V)$ denote the canonical projection. Since $p_{0} \circ D=d \circ p_{0}$, $p_{0}$ is a chain map. Obviously $p_{0} \circ \mathbf{L}=\mathrm{id}_{\mathscr{C}(F, V)}$, thus if $\mathbf{L}$ is a chain map, we obtain $\left[p_{0}\right]$ $\circ[\mathbf{L}]=\mathrm{id}_{H^{*}(P, V)}$ and $[\mathbf{L}]$ is injective. Indeed we find the following.

Proposition VI.4:

(1) $\mathbf{L}$ is a chain map and induces an injective homomorphism

$$
[\mathbf{L}]: H^{*}(F, V) \rightarrow H_{D}^{*}(\mathscr{A}(F) \otimes \operatorname{Alt}(\mathfrak{g}, V)) .
$$

(2) $L_{\bullet}^{*}$ is a chain map and thus induces a homomorphism

$$
\left[L_{\bullet}^{*}\right]: H^{*}(F, V) \rightarrow H_{l}^{*}(\mathfrak{g}, V) .
$$

Proof:

(1) By Lemma VI.2 we have

$$
\begin{aligned}
D(\mathbf{L} \omega) & =\sum_{i=0}^{n} D\left(L_{\bullet}^{i} \omega_{n}\right)=\sum_{i=0}^{n}\left[\delta_{i} L_{\bullet}^{i} \omega_{n}+(-1)^{i} d L_{\bullet}^{i} \omega_{n}\right] \\
& =\sum_{i=0}^{n}\left[L_{\bullet}^{i+1} d \omega_{n}+(-1)^{i} d L_{\bullet}^{i+1} \omega_{n}+(-1)^{i} d L_{\bullet}^{i} \omega_{n}\right] \\
& =\sum_{i=0}^{n}\left(L_{\bullet}^{i+1} d \omega_{n}\right)+(-1)^{n} d L_{\bullet}^{n+1} \omega_{n}+d \omega_{n} \\
& =\mathbf{L}\left(d \omega_{n}\right)
\end{aligned}
$$

since $L_{\bullet}^{n+1} \omega_{n}=0$.

(2) follows from Lemma VI.2 if we put $i=n+1$.

Finally we find the following result with regard to our purposes: 
Theorem VI.5: If the Lie algebra $\mathfrak{g}$ is semisimple and the form $\omega \in \mathscr{C}_{2}(F, V)_{\mathrm{inv}}$ is closed, then there exists a unique $\chi \in \mathscr{b}_{0}\left(F, \operatorname{Alt}_{1}(\mathfrak{g}, V)\right)_{\text {equiv }}$, such that

$$
d \chi=-L_{\bullet} \omega \text { and } \delta \chi=L_{\bullet}^{2} \omega .
$$

Proof: By Lemma VI.2, $\delta L_{\bullet}^{2} \omega=0$ holds. Since $H_{o}^{2}(\mathfrak{g}, V)=0$ by Whitehead's second lemma, we find $\chi \in \mathscr{C}_{0}\left(F, \operatorname{Alt}_{1}(\mathfrak{g}, V)\right)_{\text {equiv }}$ with $\delta \chi=L_{\bullet}^{2} \omega$. Lemma VI.1 yields that $\delta_{1}$ is injective, so $\chi$ is unique. On the other hand we know from $D \mathbf{L} \omega=\mathbf{L} d \omega=0$ that $-\delta L_{\bullet} \omega=d L_{\bullet}^{2} \omega=d \delta \chi=\delta d \chi$. Thus $d \chi+L_{\bullet} \omega \in \operatorname{ker} \delta_{1}$. But $\delta_{1}$ is injective.

\section{G-TRANSGRESSIVE $N$-FORMS FOR $N \leqslant 2$}

Now we are prepared to compute which closed invariant $n$-forms $\phi_{n}, n \leqslant 2$, on the fiber are $G$-transgressive.

$d \phi_{0}=0$ means that $\phi_{0} \in C^{\infty}(F)$ is locally constant. Obviously $L_{\bullet}^{\vee} \phi_{0}=0$. So every closed $G$-invariant $\phi_{0} \in C^{\infty}(F)$ is $G$-transgressive. Since $\phi_{0}$ is invariant, it is global and vertical. Thus $\left(\phi_{0}^{A}\right)^{\alpha}=\pi_{\alpha}^{\star} \phi_{0}$ and $\left[i_{\alpha, x}^{\star}\right]\left[\phi_{0}^{A}\right]=\left[\phi_{0}\right]$. This proves the following.

Lemma VII.1: Every closed $G$-invariant $\phi_{0} \in C^{\infty}(F)$ is $G$-transgressive and thus for any $\alpha$ $\in A$ and $x \in U_{\alpha},\left[i_{\alpha, x}^{\star}\right]: H^{0}(B(M, F, G)) \rightarrow H_{\mathrm{inv}}^{0}(F)$ is surjective.

[Note that this also implies $H_{\text {inv }}^{0}(F) \leqslant H^{0}(F)$, if we put $B:=\{x\} \times F$, but this is nothing new.]

For $n=1$ and $\phi_{1} \in \mathscr{b}(F)_{\text {inv }}$, Lemma VI.2 yields that $d \phi_{1}=0$ implies $\mathbf{d}_{1}^{o} L \bullet \phi_{1}=0$, i.e., for all $f \in F,\left[L \bullet \phi_{1}(f)\right] \in[\mathfrak{g}, \mathfrak{g}]^{\perp}$ by Lemma VI.1. Thus for a semisimple Lie algebra $\mathfrak{g}, L \bullet \phi_{1}=0$. As a consequence for any bundle $B(M, F, G)$ that comes along with $L,\left\{\pi_{\alpha}^{\star} \phi_{1}\right\}_{\alpha \in A}$ defines a global vertical form on $B$. We have proved the following.

Lemma VII.2: If $L$ is a Lie group action of a semisimple Lie group $G$ on $F$, then every closed invariant 1 -form $\phi_{1} \in \mathscr{b}_{1}(F)_{\text {inv }}$ is $G$-transgressive and defines a unique cohomology class $\left[\phi_{1} v\right]=\left[\left\{\pi_{\alpha}^{\star} \phi_{1}\right\}_{\alpha \in A}\right] \in H^{1}(B)$ for any bundle $B(M, F, G)$ that comes along with $L$. Thus for any $x \in U_{\alpha},\left[i_{\alpha, x}^{\star}\right]: H^{1}(B(M, F, G)) \rightarrow H_{\mathrm{inv}}^{1}(F)$ is surjective.

To show that the condition " $G$ semisimple" is necessary, take $G=\mathrm{S}^{1} \cong \mathbb{R} / \mathbb{Z}$ acting on itself by left multiplication, thus $\mathfrak{g}=\mathrm{R}$. For every Lie group, the (left) canonical 1 -form $\Theta^{L}$ $\in \mathscr{C}_{1}(G, \mathfrak{g})$, defined by $\Theta_{g}^{L}\left(\mathscr{C}_{g}\right):=d \lambda_{g^{-1}}\left(\mathscr{C}_{g}\right)$, is (left) invariant by definition. Since $S^{1}$ is Abelian, $d \Theta^{L}=0$ in this case. $\Theta^{L}$ is the volume form on $S^{1}$ and generates $H_{\text {inv }}^{1}\left(\mathrm{~S}^{1}\right) \cong H^{1}\left(\mathrm{~S}^{1}\right) \cong \mathbb{R}$, cf. Proposition IV.2. Yet $\left(L_{\bullet} \Theta^{L}\right)(X)=\Theta^{L}\left(\mathscr{L}_{X}\right)=X$ for all $X \in R$. Thus $L_{\bullet} \Theta^{L}=\mathrm{id}_{\mathrm{R}}$ and $\Theta^{L}$ is not $\mathrm{S}^{1}$-transgressive.

In fact, take the principal bundles $P_{m}\left(\mathrm{~S}^{2}, \mathrm{~S}^{1}\right), m \in \mathbb{Z}$, that classify all fiber bundles over $\mathrm{S}^{2}$ with structure group $\mathrm{S}^{1}$ according to the Classification theorem (Ref. 4, p. 99). For $m=0$ we have the trivial bundle $S^{2} \times S^{1}$ and for $m=1$ we obtain the Hopf fibering of the 3-sphere, $\pi: S^{3} \rightarrow S^{2}$. For the de Rham cohomology $H^{*}\left(P_{m}\right)$ one obtains from the spectral sequence for $P_{m}$ with $m \neq 0$ :

$$
H^{0}\left(P_{m}\right) \cong \mathrm{R}, \quad H^{1}\left(P_{m}\right)=0, \quad H^{2}\left(P_{m}\right)=0, \quad H^{3}\left(P_{m}\right) \cong \mathrm{R} .
$$

So no $\left[i_{\alpha, x}^{\star}\right]: H^{1}\left(P_{m}\right) \rightarrow H_{\text {inv }}^{1}(G)$ is surjective. Moreover, we always have $\Theta^{L} v=\omega^{\Gamma}$, even for $m=0$. Since $d \omega^{\Gamma}=d^{\Gamma} \omega^{\Gamma}=\Omega^{\Gamma}$, our canonical construction does not produce closed forms on $P_{m}$, in general.

Finally we consider the case $n=2$ for semisimple Lie groups. Using Theorem VI.5 we obtain that every closed invariant 2 -form on $F$ is $G$-transgressive. Thus we have the following.

Corollary VII.3: If $L$ is a Lie group action of a semisimple Lie group $G$ on $F$, then every closed invariant 2-form $\phi_{2} \in \mathscr{A}_{2}(F)_{\text {inv }}$ is $G$-transgressive and defines a unique cohomology class $\left[\phi_{2}^{A}\right] \in H^{2}(B) \quad$ for any bundle $B(M, F, G)$ that comes along with L. If $\chi_{0}^{1}$ $\in C^{\infty}(F)_{\text {equiv }} \otimes \operatorname{Hom}(\mathfrak{g}, \mathbb{R})$ is the unique map with $d \chi_{0}^{1}=-L_{\bullet} \phi_{2}$ and $\delta \chi_{0}^{1}=L_{\bullet}^{2} \phi_{2}$ according to Theorem VI.5, then $\phi_{2}^{A}$ is given by 


$$
\phi_{2}^{A}=\phi_{2} v+\left(\chi_{0}^{1} v\right) \cdot \mathrm{F} \in \mathscr{C}_{2}(B)
$$

Thus for any $x \in U_{\alpha},\left[i_{\alpha, x}^{\star}\right]: H^{2}(B(M, F, G)) \rightarrow H_{\mathrm{inv}}^{2}(F)$ is surjective.

In view of Proposition IV.2 we thus have proved the following.

Theorem VII.4: If $L$ is a Lie group action of a semisimple Lie group $G$ on $F$, then every closed invariant $\phi_{n} \in \mathscr{A}_{n}(F)_{\mathrm{inv}}, n \leqslant 2$, is $G$-transgressive and defines a unique cohomology class $\left[\phi_{n}^{A}\right] \in H^{n}(B)$ for any bundle $B(M, F, G)$ that comes along with $L$. For any $x \in U_{\alpha}$, $\left[i_{\alpha, x}^{\star}\right]: H^{n}(B(M, F, G)) \rightarrow H_{\mathrm{inv}}^{n}(F)$ is surjective.

If in addition, $G$ is compact and connected, then $H_{\text {inv }}^{n}(F) \cong H^{n}(F)$. Thus for every bundle $B(M, F, G), H^{n}(B)$ contains a subgroup isomorphic to $H^{n}(F)$ for $n \leqslant 2$.

Theorem VII.4 applies to QCD, where $G \cong \mathrm{SU}_{3}$ and to Yang-Mills theories with $G<\mathrm{SU}_{n} \times \mathrm{SU}_{n}$.

This theorem is sharp in the sense that it does not hold for $n=3$, e.g., take $G=\mathrm{S}^{3} \cong \mathrm{SU}_{2}$ acting on itself by left multiplication. Then the volume form on $\mathrm{S}^{3}$ is closed and invariant and generates $H^{3}\left(\mathrm{~S}^{3}\right) \cong \mathrm{R}$. If this form were $G$-transgressive, then for all principal bundles $P\left(M, \mathrm{~S}^{3}\right)$, the cohomology group $H^{3}(P)$ would contain a subgroup isomorphic to $\mathbb{R}$, independently of $M$ and the transition functions $g_{\alpha \beta}$. Yet we know that $\mathrm{S}^{7}$ is a principal bundle over $\mathrm{S}^{4}$ with fiber $\mathrm{S}^{3}$, and $H^{3}\left(\mathrm{~S}^{7}\right)=0$. Thus the volume form on $\mathrm{S}^{3}$ cannot be $G$-transgressive.

\section{FINAL REMARKS}

According to Corollary IV.8, every $G$-transgressive form is 0 -transgressive for all bundles with fiber and left action $L$. The reverse is also true for compact connected Lie groups. This can be proved, e.g., in terms of universal bundles, the Weil algebra and the equivariant cohomology of the $G$-manifold $F{ }^{9,10}$ In fact, $G$-transgressive forms are exactly those forms on $F$ that generate equivariant cohomology classes (but not every equivariant cohomology class is generated by a form on $F$ ). Now there is a natural isomorphism between this equivariant cohomology and the de Rham cohomology of the universal bundle for the given left action $L$, which yields that equivariant cohomology classes define de Rham cohomology classes on all bundles that come with $L$.

We have not used these notions here for several reasons: First of all, we did not want to restrict ourselves a priori to compact connected Lie groups, where equivariant cohomology is usually settled. Second, we were not interested in the whole cohomology of the bundles (resp., the whole equivariant cohomology), but only in those cohomology classes that have their origin in forms on $F$. And last, for the applications in theoretical physics, we were interested in explicit formulas for the generated differential forms and not in a more abstract notion like the Weil algebra.

For non-compact Lie groups the reverse of Corollary IV.8 is false, e.g., take $G=\mathbb{R}$ and define $L: \mathbb{R} \times \mathbb{R}^{k} \rightarrow \mathbb{R}^{k}$ by $L(r, \vec{v})=\vec{v}+r \vec{z}$ with $\vec{z} \in \mathbb{R}^{k}$. Then all forms $\phi_{n}$ with constant coefficients are closed and invariant. Because every bundle with structure group $\mathbb{R}$ (even more general, with $\left.G \cong \mathbb{R}^{m}\right)$ is trivial, every $\phi_{n}$ defines a closed form $\operatorname{pr}_{\mathrm{R}^{k}}^{\star} \phi_{n}$ on the bundle. But $\phi_{n}$ is not $G$-transgressive, in general, e.g., for $\phi_{1} \in \mathscr{C}_{1}\left(\mathbb{R}^{k}\right)$ defined by $\phi_{1}(\vec{v})(\vec{x}):=\langle\vec{v}, \vec{z}\rangle$ for all $\vec{x} \in \mathbb{R}^{k}$ and $\vec{v} \in T_{\vec{x}}\left(\mathrm{R}^{k}\right)$, where $L_{\bullet} \phi_{1}(\vec{x})=\mathrm{id}_{\mathrm{R}} \neq 0$. Thus $\phi_{1}$ is not $G$-transgressive.

\footnotetext{
${ }^{1}$ T. H. R. Skyrme, “A unified field theory of mesons and baryons," Nucl. Phys. 31, 556-569 (1962).

${ }^{2}$ E. Witten, "Global aspects of current algebra," Nucl. Phys. B 223, 422-432 (1983).

${ }^{3}$ I. Zahed and G. E. Brown, “The Skyrme model," Phys. Rep. 142, (1 \& 2), 1-102 (1986).

${ }^{4}$ N. Steenrod, The Topology of Fibre Bundles (Princeton University, Princeton, NJ, (1951).

${ }^{5}$ S. Kobayashi and K. Nomizu, Foundations of Differential Geometry (Wiley, New York, 1963), Vol. I.

${ }^{6}$ R. Bott and L. W. Tu, Differential Forms in Algebraic Topology (Springer-Verlag, New York, 1982).

${ }^{7}$ C. Gross, "Connections on fiber bundles and canonical extensions of differential forms," Preprint No. 1795 at: http:// www.mathematik.th-darmstadt.de/prepr.

${ }^{8}$ C. Gross, "Operators on differential forms for Lie transformation groups," J. Lie Theory 6, 1-17 (1996).

${ }^{9}$ M. F. Atiyah and R. Bott, “"The moment map and equivariant cohomology," Topology 23, 1-28 (1984).
} 
${ }^{10}$ V. Mathai and D. Quillen, "Superconnections, Thom classes, and equivariant forms," Topology 25, 85-110 (1986).

${ }^{11}$ C. E. Callan and E. Witten, "Monopole catalysis of skyrmion decay," Nucl. Phys. B 239, 161-176 (1984).

${ }^{12}$ M. Chemtob, "CCross section of monopole-induced skyrmion decay," Phys. Rev. D 39, 2013-2031 (1989).

${ }^{13}$ C. Gross, "Topology of the skyrmion bundle,"' J. Math. Phys. 36, 4406-4435 (1995).

${ }^{14} \mathrm{~W}$. Greub, S. Halperin, and R. Vanstone, Connections, Curvature, and Cohomology (Academic, New York, 1972), Vol. I-III.

${ }^{15}$ Ö. Kaymakcalan, S. Rajeev, and J. Schechter, “Non-Abelian anomaly and vector-meson decays,” Phys. Rev. D 30, 594-602 (1984).

${ }^{16}$ N. K. Pak and P. Rossi, "Gauged Goldstone boson effective action from direct integration of Bardeen anomaly," Nucl. Phys. B 250, 279-294 (1985).

${ }^{17} \mathrm{C}$. Gross, "The $D_{2}$-operator of the spectral sequence for $\mathrm{S}^{1}$-bundles," (submitted).

${ }^{18}$ J. Hilgert and K.-H. Neeb, Lie-Gruppen und Lie-Algebren (Vieweg, Braunschweig, 1991). 